\title{
Simulation of asymmetric Lamb waves for sensing and actuation in plates
}

\author{
A. Ghoshal ${ }^{\mathrm{a}}$, W.N. Martin ${ }^{\mathrm{b}}$, M.J. Schulz ${ }^{\mathrm{c}}$, W.H. Prosser ${ }^{\mathrm{d}}$ and A. Chattopadhyay ${ }^{\mathrm{e}}$ \\ ${ }^{a}$ Structural Integrity Group, United Technologies Research Center, East Hartford, CT 06108 (formerly National \\ Research Council Associate, NASA Langley Research Center, Hampton, VA, USA \\ E-mail: anindo ghoshal@yahoo.comor ghoshaa@utrc.utc.com \\ ${ }^{\mathrm{b}}$ Naval Undersea Warfare Center, Newport, RI 02841, USA \\ E-mail: MartinWN@Npt.NUWC.Navy.Mil \\ ${ }^{\mathrm{c}}$ Associate. Department of Mechanical Engineering, University of Cincinnati, OH 45221, USA \\ E-mail:mark.j.schulz@uc.edu \\ ${ }^{\mathrm{d}}$ MS 231, NESB, 3B Taylor St, NASA Langley Research Center, Hampton, VA 23681, USA \\ E-mail: William.H.Prosser@nasa.gov \\ ${ }^{\mathrm{e}}$ Department of Mechanical Engineering and Aerospace Engineering, Arizona State University, Tempe, AZ 85287, \\ USA \\ E-mail: aditi@asu.edu
}

Received 20 August 2003

Revised 21 June 2004

\begin{abstract}
Two approaches used for monitoring the health of thin aerospace structures are active interrogation and passive monitoring. The active interrogation approach generates and receives diagnostic Lamb waves to detect damage, while the passive monitoring technique listens for acoustic waves caused by damage growth. For the application of both methods, it is necessary to understand how Lamb waves propagate through a structure. In this paper, a Physics-Based Model (PBM) using classical plate theory is developed to provide a basic understanding of the actual physical process of asymmetric Lamb mode wave generation and propagation in a plate. The closed-form model uses modal superposition to simulate waves generated by piezoceramic patches and by simulated acoustic emissions. The generation, propagation, reflection, interference, and the sensing of the waves are represented in the model, but damage is not explicitly modeled. The developed model is expected to be a useful tool for the Structural Health Monitoring (SHM) community, particularly for studying high frequency acoustic wave generation and propagation in lieu of Finite Element models and other numerical models that require significant computational resources. The PBM is capable of simulating many possible scenarios including a variety of test cases, whereas experimental measurements of all of the cases can be costly and time consuming. The model also incorporates the sensor measurement effect, which is an important aspect in damage detection. Continuous and array sensors are modeled, which are efficient for measuring waves because of their distributed nature.
\end{abstract}

\section{Introduction}

Active propagation of Lamb waves is an emerging technique used to detect damage [1-7] in aerospace structures. Passive methods of measuring Acoustic Emissions (AE) are also used for damage detection [8-12]. The modeling of wave propagation for these damage detection techniques is often conducted for materials that are of infinite lateral dimension [13-18,27] because closed-form solutions of wave propagation in a bounded medium are difficult to obtain. However, a normal mode approach for obtaining a closed form solution for bending wave propagation in a finite plate is presented in [19-22]. Finite-element methods are also used to model wave propagation in 


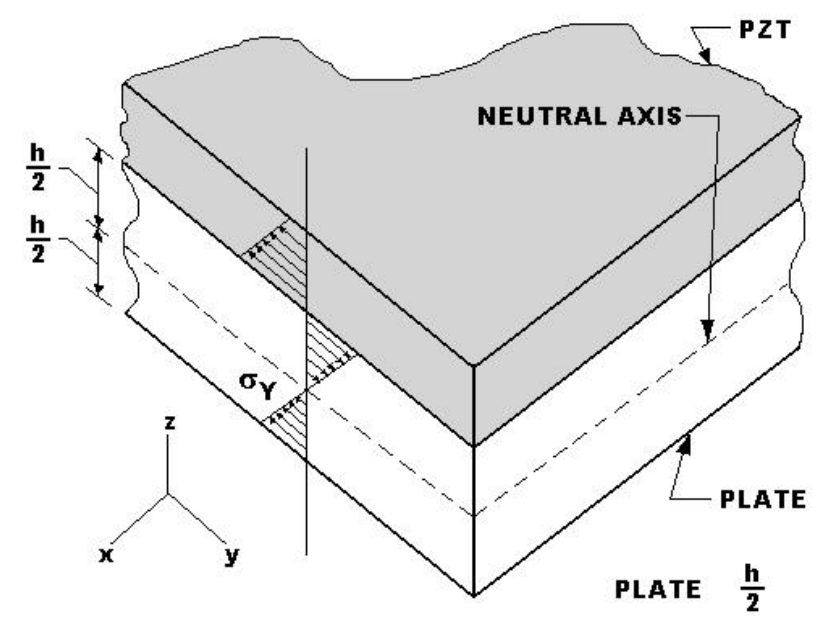

Fig. 1. Lateral sides of an element of the PZT with the plate underneath.

bounded media, but the computation time is usually large and the representation of higher frequencies and modes are constrained by the number of elements used.

The normal mode approach is also used in the present paper to model asymmetric Lamb wave or flexural wave propagation in a simply supported plate. Previously performed work [19-22] suggests that cracks generate predominantly extensional mode AE signals and to a lesser extent flexural mode AE signals. Out of plane sources like impact damage or delamination generate large flexural modes. Cracks not located at the mid-plane of a plate will also generate flexural modes, but cracks located near the mid-plane generate predominately symmetric modes. The asymmetric waves usually produce larger responses from sensors located on the surface of the plate, which is the simplest and possibly most practical application of sensors for SHM. Hence the present simulated AE model focuses on flexural mode AE signals generated from impact damage, delaminations, and cracks not located at the mid-plane, and due to active excitations.

A glass epoxy composite plate is modeled here as quasi-isotropic, and piezoceramic Lead Zirconate Titanate (PZT) actuators and sensors are modeled on the plate for generating and receiving the waves. The objective of developing this model is to provide a tool to simulate wave propagation and to facilitate the design of sensors to measure the waves. The model uses a closed-form solution and it is written in matrix and vector forms to run efficiently on a stand-alone PC. With this technique, series and array configurations of sensors can be investigated including different shapes of sensors and actuators. Piezoelectric patches are used as the sensor and actuator material. Modeling of PZT actuators bonded to structures is based upon references [23-26].

The advantage of this technique in contrast to finite element methods is the ability to simulate waves using much less computational time. Numerical results of the sensor outputs are presented in the simulated cases. The principal goal of the present research is the development of the PBM of the acoustic wave generation, propagation and sensing in simply supported plates, based on the classical vibration equations of motion. Details of the continuous sensor array and the cross-sensor array are also based on references [27,31].

\section{Asymmetric Lamb wave modeling}

Modeling of acoustic wave generation and propagation in plates is discussed in the following sections. The acoustic waves in plates are generated passively using a mechanical pencil lead break input, and actively using a surface bonded piezoelectric actuator. To model impact, delamination, or crack propagation, a transient excitation such as a delta or step function is needed. For active wave propagation, an impulse or sine tone burst can be used. 


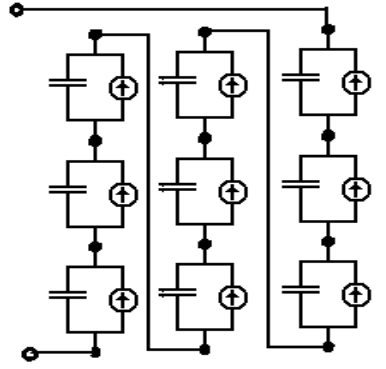

(a)

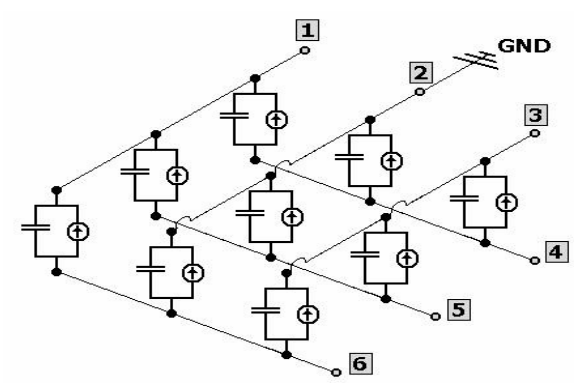

(b)

Fig. 2. Circuit model of sensors showing (a) a continuous sensor array and (b) a cross-sensor array.

\subsection{Acoustic emission modeling}

The following is the equation of motion for an isotropic plate subject to a step load excitation at an arbitrary location on the plate surface. Quasi-isotropic materials can be approximated using this equation. Such a step force load is representative of a transient acoustic emission source from propagating damage and is known as a Hsu-Nielsen source. The equation of motion is:

$$
\mathrm{D} \nabla^{4} \mathrm{w}(\mathrm{x}, \mathrm{y}, \mathrm{t})=-\rho \mathrm{h} \ddot{w}+f(x, y, t)
$$

where the step function force $f(x, y, t)=f_{0} U(t) \delta\left(x-x_{c}\right) \delta\left(y-y_{c}\right)$ gives the magnitude of the input excitation, $\mathrm{w}$ is the z-displacement, $\rho$ is mass density and $h$ is the plate thickness. The plate flexural rigidity is given as: $D=\underset{12\left(1-\nu^{2}\right)}{E h^{3}}$, and $\mathrm{U}(\mathrm{t})=0$ when $\mathrm{t} \leqslant 0$ and $U(t)=1$ when $t>0$. The quantities $x_{c}$ and $y_{c}$ denote the coordinates of the location of lead break on the plate. In previous studies, a source amplitude of $f_{0}=1 \mathrm{~N}$ [19-22] was used to model a $0.3 \mathrm{~mm}$ pencil lead break and the same is assumed in the present work. In general, $f(x, y, t)$ is considered to be the normal component of all of the body forces or a concentrated normal surface force. The solution of Eq. (1) is obtained by applying the Navier solution [29] and by using the orthogonalization property for separation of the spatial and time variables. The temporal equation thus obtained can be solved for each mode. A detailed derivation for an impulse input excitation on a simply supported plate is presented in [11]. The detailed derivation shown in Appendix B considers the step input.

Thus the total solution for the plate displacement as shown in Appendix B can be expressed as:

$$
w(x, y, t)=\sum_{n} \sum_{m}\left(\begin{array}{c}
F_{m n} \\
\omega_{m n}^{2}
\end{array}-\left(\begin{array}{c}
F_{m n} \\
\omega_{m n} \omega_{d_{m n}}
\end{array} e^{-\zeta_{m n} \omega_{m n} t} \cos \left(\omega_{d_{m n}} t-\theta\right)\right)\right) \sin \left(\begin{array}{c}
m \pi x \\
a
\end{array}\right) \sin \left(\begin{array}{c}
n \pi y \\
b
\end{array}\right)
$$

The damping ratio is $\zeta_{m n}$ and can be specified individually for each mode. For zero damping, i.e., when $\zeta=0$,

$$
w(x, y, t)=\sum_{n} \sum_{m} F_{m n}^{2}\left(1-\cos \left(\omega_{m n} t\right)\right) \sin \left(\begin{array}{c}
m \pi x \\
a
\end{array}\right) \sin \left(\begin{array}{c}
n \pi y \\
b
\end{array}\right)
$$

The corresponding strains can be written as:

$$
\begin{aligned}
& \varepsilon_{x}(x, y, t)=-z \frac{\partial^{2} w}{\partial x^{2}}=-z \sum_{n} \sum_{m} a_{m n}(t) \sin \left(\begin{array}{c}
m \pi x \\
a
\end{array}\right) \sin \left(\begin{array}{c}
n \pi y \\
b
\end{array}\right)\left(\begin{array}{c}
m \pi \\
a
\end{array}\right)^{2} \\
& \varepsilon_{y}(x, y, t)=-z \sum_{n} \sum_{m} a_{m n}(t) \sin \left(\begin{array}{c}
m \pi x \\
a
\end{array}\right) \sin \left(\begin{array}{c}
n \pi y \\
b
\end{array}\right)\left(\begin{array}{c}
n \pi \\
b
\end{array}\right)^{2} \\
& \gamma_{x y}(x, y, t)=2 z \begin{array}{c}
\partial^{2} w \\
\partial x \partial y
\end{array}=2 z \sum_{n} \sum_{m} a_{m n}(t) \cos \left(\begin{array}{c}
m \pi x \\
a
\end{array}\right) \cos \left(\begin{array}{c}
n \pi y \\
b
\end{array}\right)\left(\begin{array}{c}
m \pi \\
a
\end{array}\right)\left(\begin{array}{c}
n \pi \\
b
\end{array}\right)
\end{aligned}
$$




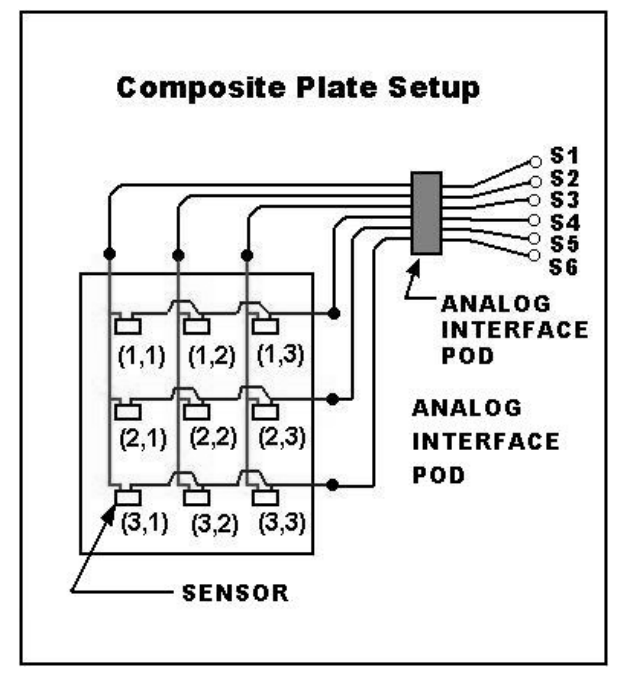

Fig. 3. Schematic view of the sensor placement on the composite plate.

where the $\varepsilon_{x}$ and $\varepsilon_{y}$ are the in-plane strains and $\gamma_{x y}$ is the shear strain. The average strains over the area of the sensor are used to compute the voltage output from the PZT sensors used in this work. For a simple approximation to model a unidirectional Active Fiber Composite patch bonded to a plate, the open circuit output voltage can be approximated using the average strain over the sensor nodes as follows:

$$
V_{0}=\bar{\varepsilon} h e / \varepsilon^{s}
$$

where $\varepsilon$ is the average strain and $\varepsilon^{s}$ is the permittivity. The average strain in the $X$-direction is computed for the nodes as:

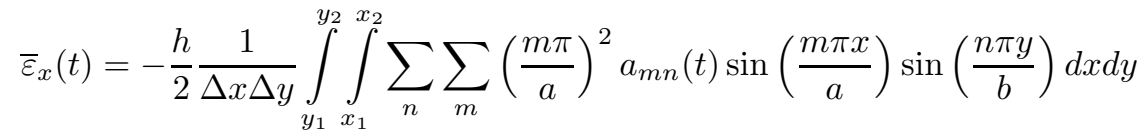

Integrating, the average strain in the $\mathrm{x}$-direction is expressed as:

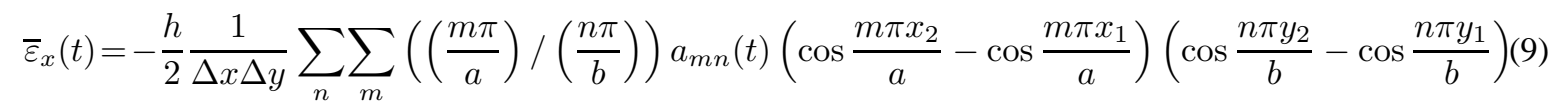

where $\Delta x=x_{2}-x_{1}$ and $\Delta y=y_{2}-y_{1}$. The strain in the $y$-direction is computed similarly. The corresponding strain equations with damping can be derived similarly. The combination of the strains in both directions using bi-axial stress-strain equations gives the total strain. In the numerical simulation, the elastic solution is computed at small time steps. The solution procedure requires that a sufficient number of modes are used in the superposition solution such that the modeled rate of change of the excitation is less than the speed of the propagating waves in the material. Using this solution, single and continuous sensor designs can be practically studied to sense AE signatures and wave propagation. This particular AE model is valid for simply supported plates and such a closed form solution may not be possible for clamped boundary conditions. For other boundary conditions, one may have to use a different displacement function or coefficients as in a Rayleigh-Ritz approximate model. However, the simply-supported model gives a very good understanding of how asymmetric Lamb waves are generated and propagate through a plate and how they may be applied for applications like SHM.

\subsection{Equivalent circuit of the PZT patch}

The PZT patches are convenient for use in SHM and Active Control. They can be modeled as a capacitor in parallel with a current source because of the piezoelectric properties of the sensors. The piezoelectric constitutive equations, as listed in the IEEE standard ANSI/IEEE Standard 176-1987, can be used to show the basis of the PZT 

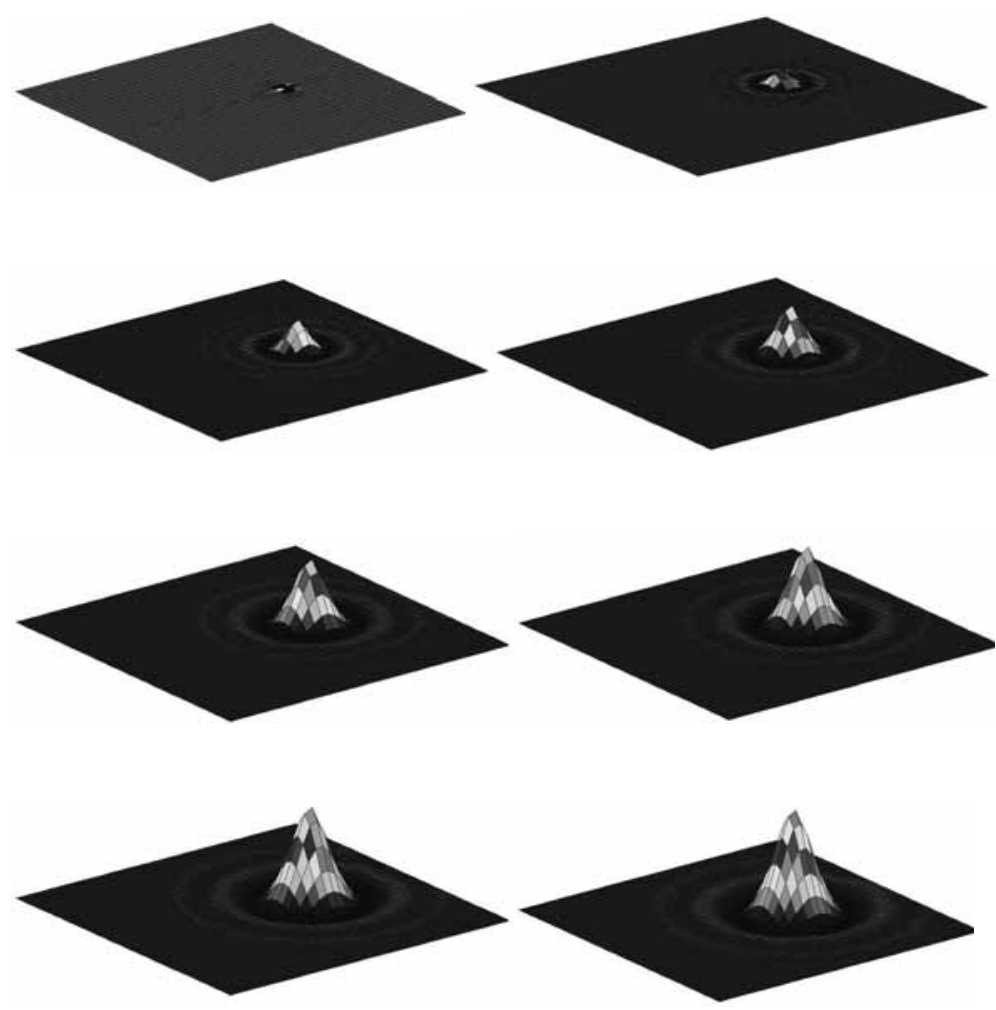

Fig. 4. Acoustic Wave propagation at 10,60, 110, 160, 210, 260, 310, 360 micro-sec due to a lead break excitation modeled as a step input function.

electrical modeling. The following derivations assume a monolithic PZT element, and ideal transfer of strain to the element. The matrix form of the piezoelectric constitutive equations can be expressed as:

$$
\begin{aligned}
& {\left[\begin{array}{l}
D \\
T
\end{array}\right]=\left[\begin{array}{ll}
\varepsilon^{S} & e \\
-e_{t} & c^{E}
\end{array}\right] \cdot\left[\begin{array}{l}
E \\
S
\end{array}\right]} \\
& {\left[\begin{array}{c}
D \\
S
\end{array}\right]=\left[\begin{array}{ll}
\varepsilon^{T} & d \\
d_{t} & s^{E}
\end{array}\right] \cdot\left[\begin{array}{l}
E \\
T
\end{array}\right]}
\end{aligned}
$$

In these relations, $\varepsilon^{S}$ is a 3 by 3 matrix describing the permittivity components, $\varepsilon_{i j}$ (farads/m) under a constant strain condition. The superscript $\mathrm{T}$ indicates a constant stress condition and the subscript $\mathrm{t}$ means transposed. The induced stress is a 3 by 3 matrix of the components $\mathrm{e}_{i j}\left(\mathrm{Coulomb} / \mathrm{m}^{2}\right)$. The piezoelectric charge coefficients comprise the elements of a 3 by 6 matrix of the components $d_{i j}(\mathrm{~m} / \mathrm{V})$. The short circuit compliance matrix is a 6 by 6 matrix with components $\mathrm{s}_{i j}^{E}\left(\mathrm{~m}^{2} / \mathrm{N}\right)$. The short circuit stiffness matrix is a 6 by 6 matrix with components $\mathrm{c}_{i j}^{E}\left(\mathrm{~N} / \mathrm{m}^{2}\right)$. The electrical displacement is a 3 by 1 vector of the components $\mathrm{D}_{i}\left(\right.$ Coulombs $\left./ \mathrm{m}^{2}\right)$. The stress is a 3 by 1 vector of the components $\mathrm{T}_{i}\left(\mathrm{~N} / \mathrm{m}^{2}\right)$. The electric field is a 3 by 1 vector of the components $\mathrm{E}_{i}(\mathrm{~V} / \mathrm{m})$ and the strain is a 3 by 1 vector with components $S_{i}(\mathrm{~m} / \mathrm{m})$.

If the electric field is only applied through one axis, then $\mathrm{D}$ and $\mathrm{E}$ are scalar values. Algebraic comparison of Eqs (10) and (11) show that the induced stress constant can be written as:

$$
e=d c^{E}
$$

and therefore can be readily calculated from the PZT properties. From Eq. (10), the electrical displacement can be written as follows:

$$
D=\varepsilon^{S} E+e S
$$




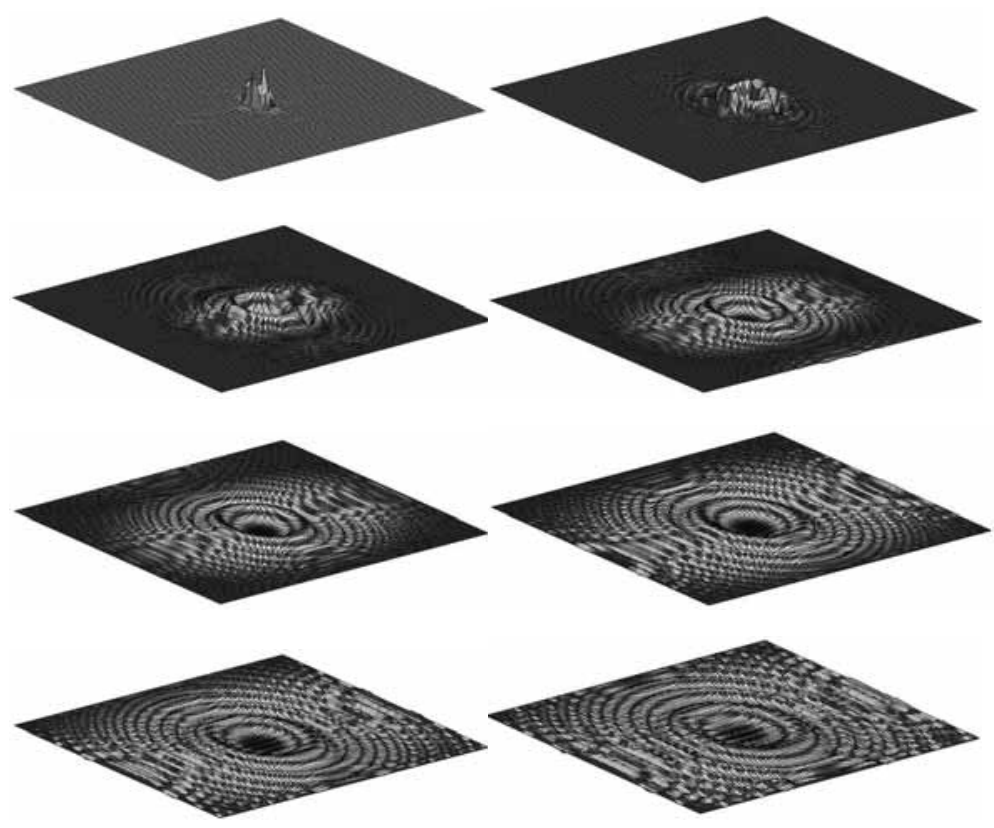

Fig. 5. Wave propagation at 10,60,110,160, 210, 260, 310, 360 micro-sec due to center actuation by a PZT patch with an impulse moment excitation.

Considering a monolithic, electroded piezoceramic material subjected to bi-directional strain, Eq. (13) becomes:

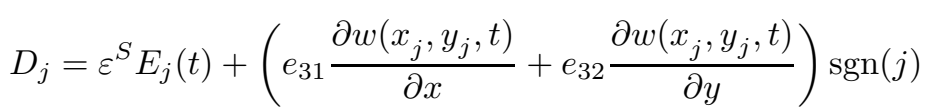

Corresponding to Eq. (14), the current generated by the $j$ th segment of the sensor is:

$$
i_{j}=\dot{D}_{j} A_{e}=C_{p} \dot{V}_{o}+\left(\begin{array}{cc}
e_{31} \partial^{2} w\left(x_{j}, y_{j}, t\right) & \partial x \partial t
\end{array}+e_{32} \begin{array}{c}
\partial^{2} w\left(x_{j}, y_{j}, t\right) \\
\partial y \partial t
\end{array}\right) A_{e} \operatorname{sgn}(j)
$$

where $j$ is used to represent the $j^{t h}$ electroded section of the sensor, $t$ is time, $w\left(x_{j}, t\right)$ is the plate displacement at segment $j$, and the $s g n$ function allows for the connection of the sensors in positive or negative polarities. The simply supported plate is modeled to study the benefits of various PZT sensor array configurations for measuring strain and acoustic emissions. The thin glass epoxy composite plate used in the experiment is modeled as quasi-isotropic.

The derivations for calculating the voltage output of PZT sensors in various configurations are described in detail by Martin et al. [27]. The aforementioned equations depend on the strain or strain rate of the PZT wafer. In this work, the PZT sensors are bonded to a simply supported plate. The simulation is used to determine the strains and displacements of the simply supported plate. The strains or strain rates from the simulation are then substituted into the necessary equations to determine the current of the sensor configuration used. For closed circuit cases, the current outputs are computed using the Newmark-beta numerical integration method. The voltage output response is then computed as the product of the current and the external resistance in the oscilloscope, which is 1-M $\Omega$, with the currents depending on the type of sensor configuration used. The sensor configurations and their outputs are included in the plate simulation program.

\subsection{Modeling active wave generation and propagation}

An analytical model has been created for active acoustic wave generation and propagation in a plate due to surface bonded PZT patches. The closed form solution for wave generation due to actuation by a PZT actuator is detailed here. From the classical theory of vibration of plates, the equation of motion has been derived in terms of internal plate flexural moments $M_{x}, M_{x y}$, and $M_{y}$, and actuator induced moments $m_{x}$ and $m_{y}$ and then expressed 

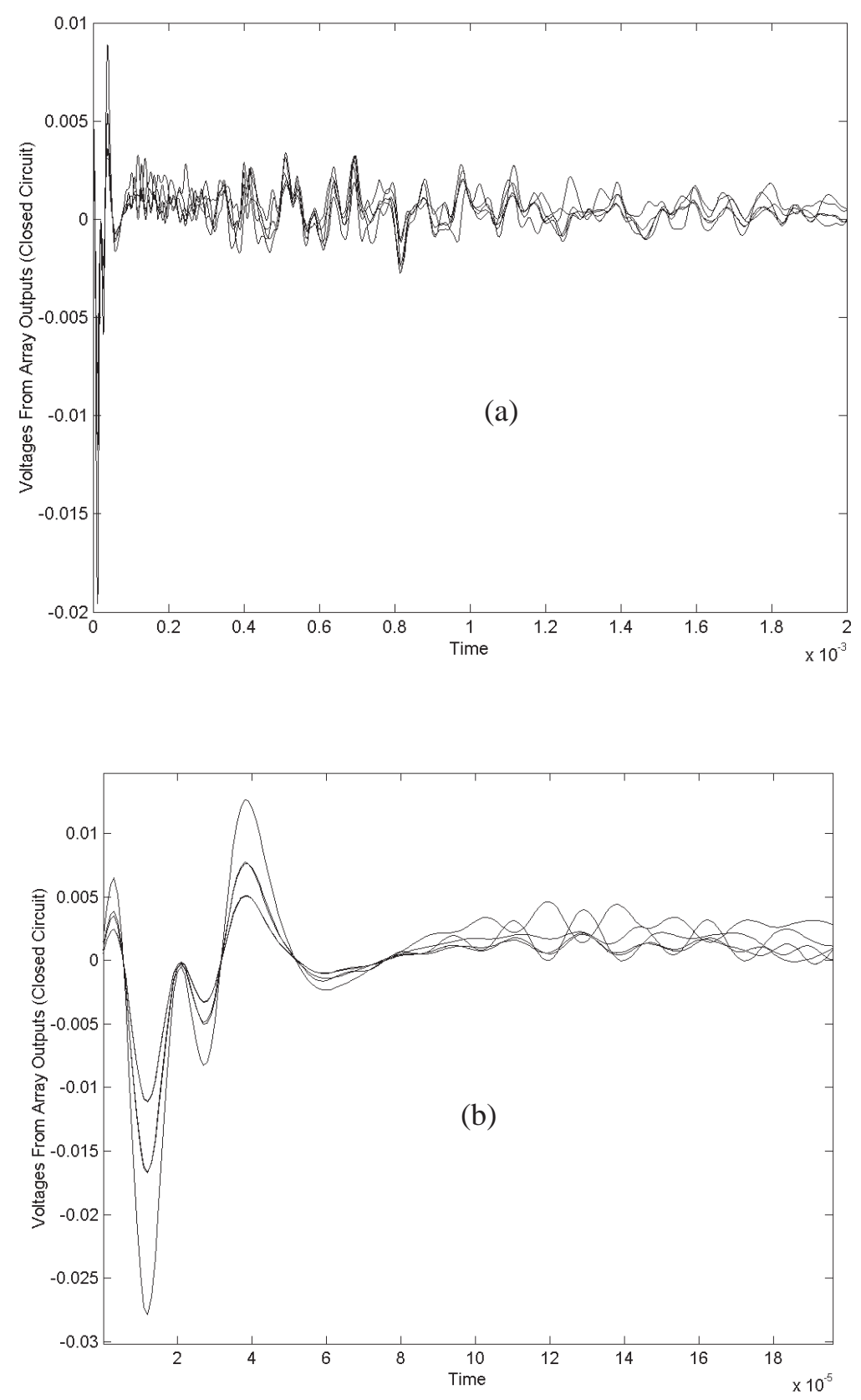

Fig. 6. Voltage time history due to center actuation by a PZT patch; (a) array voltage for $2 \mathrm{~ms}$, (b) array voltage for $0.2 \mathrm{~ms}$.

in displacement form as:

$$
D \nabla^{4} w(x, y, t)+\rho h \ddot{w}=\begin{gathered}
\partial^{2} m_{x} \\
\partial x^{2}
\end{gathered}+\begin{gathered}
\partial^{2} m_{y} \\
\partial y^{2}
\end{gathered}
$$

The moments induced by the actuator patch are expressed as Heaviside step functions. The temporal equation is derived in terms of modes using separation of spatial and time variables, and orthogonal relationships of trigonometric functions. A similar procedure was followed in the pencil lead break analysis to solve for the plate displacements 

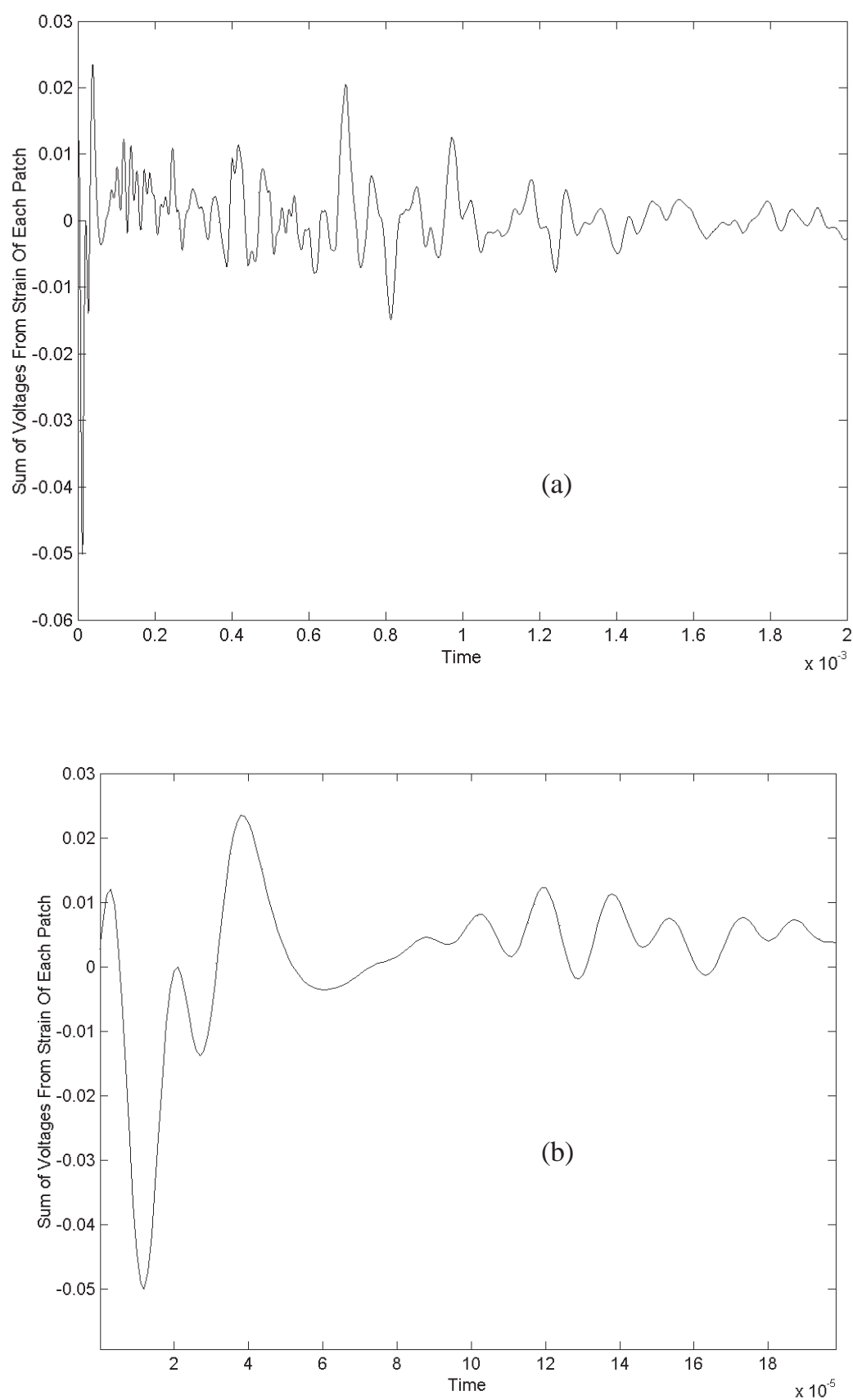

Fig. 7. Voltage time history due to center actuation by a PZT patch; (a) continuous sensor voltage for $2 \mathrm{~ms}$, (b) continuous sensor voltage for $0.2 \mathrm{~ms}$.

due to step excitation. For an impulse moment actuation the plate displacement is expressed in terms of a Fourier series (Navier's solution) and by summation of modes including damping as shown below:

$$
w(x, y, t)=\sum_{n} \sum_{m} \begin{gathered}
F_{m n} \\
\omega_{d_{m n}}
\end{gathered} e^{-\zeta_{m n} \omega_{m n} t} \sin \left(\omega_{d_{m n}} t\right) \sin \left(\begin{array}{c}
m \pi x \\
a
\end{array}\right) \sin \left(\begin{array}{c}
n \pi y \\
b
\end{array}\right)
$$




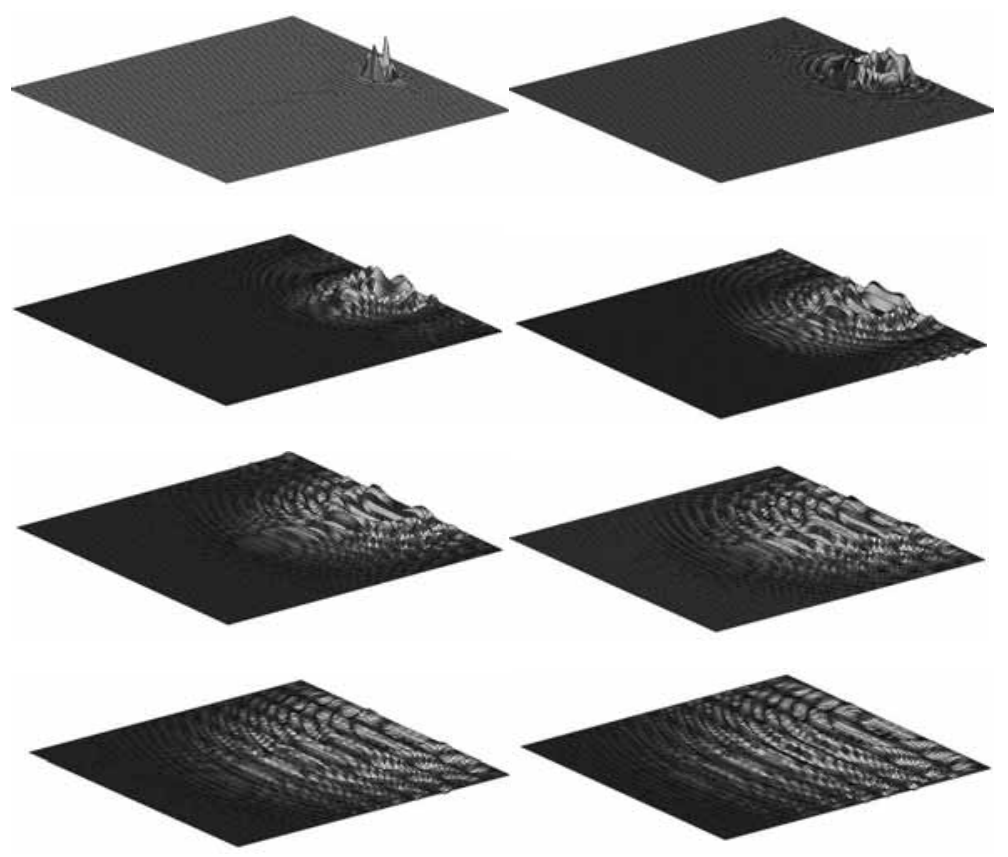

Fig. 8. Wave propagation at $10,60,110,160,210,260,310,360$ micro-sec due to edge actuation by a PZT patch.

where

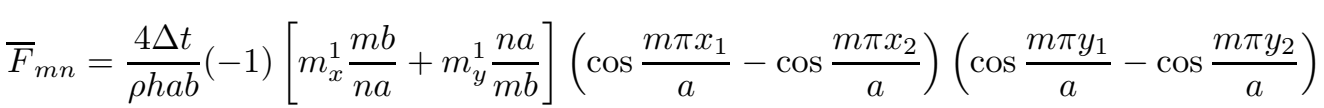

The variables $a$ and $b$ are the width and length of the plate in $x$ and $y$ directions, $m$ and $n$ are the mode numbers, $h$ is the thickness of the plate, $m_{x}^{1}$ and $m_{y}^{1}$ are the distributed surface moments, $\rho$ is the mass density of the glass-epoxy composite plate, and $z$ is $h / 2$. The corresponding strains are computed from Eqs (4-6). The variables $x_{1}, x_{2}, y_{1}$ and $y_{2}$ represent the boundaries or edges of the PZT element.

The derivation of $m_{x}^{1}$ and $m_{y}^{1}$ requires certain assumptions. The assumptions are as follows:

(i) The PZT patch element is poled in the $z$-direction and experiences equal strains in the $x-$ and $y$-directions when exposed to an electric field. This is true only if the strain coefficients $d_{31}$ and $d_{32}$ are equal which is the case in this present work.

(ii) The host plate is a rectangular simply supported isotropic plate.

(iii) The stress distribution within the plate is assumed to be symmetric about the neutral axis and the bending of the plate produces a linear normal stress distribution. Thus the neutral axis is assumed to be coincident with the mid-plane of the plate. This is a reasonable approximation because usually the mass and flexural rigidity of the PZT element are significantly less than that of the plate [25].

(iv) Actuator stress can be integrated to obtain the equivalent bending moment.

(v) The bond between the plate and the PZT element is assumed to be perfect.

(vi) The strain distribution through the thickness of the PZT element is assumed to have the same slope as the stress distribution through the thickness of the host plate.

The unconstrained strains in the PZT element due to the input actuation voltage V can be written as:

$$
\begin{gathered}
\left(\varepsilon_{x}\right)_{p z t}=\begin{array}{c}
d_{31} \cdot V \\
t_{p z t}
\end{array} \\
\left(\varepsilon_{y}\right)_{p z t}=\begin{array}{c}
d_{32} \cdot V \\
t_{p z t}
\end{array}
\end{gathered}
$$



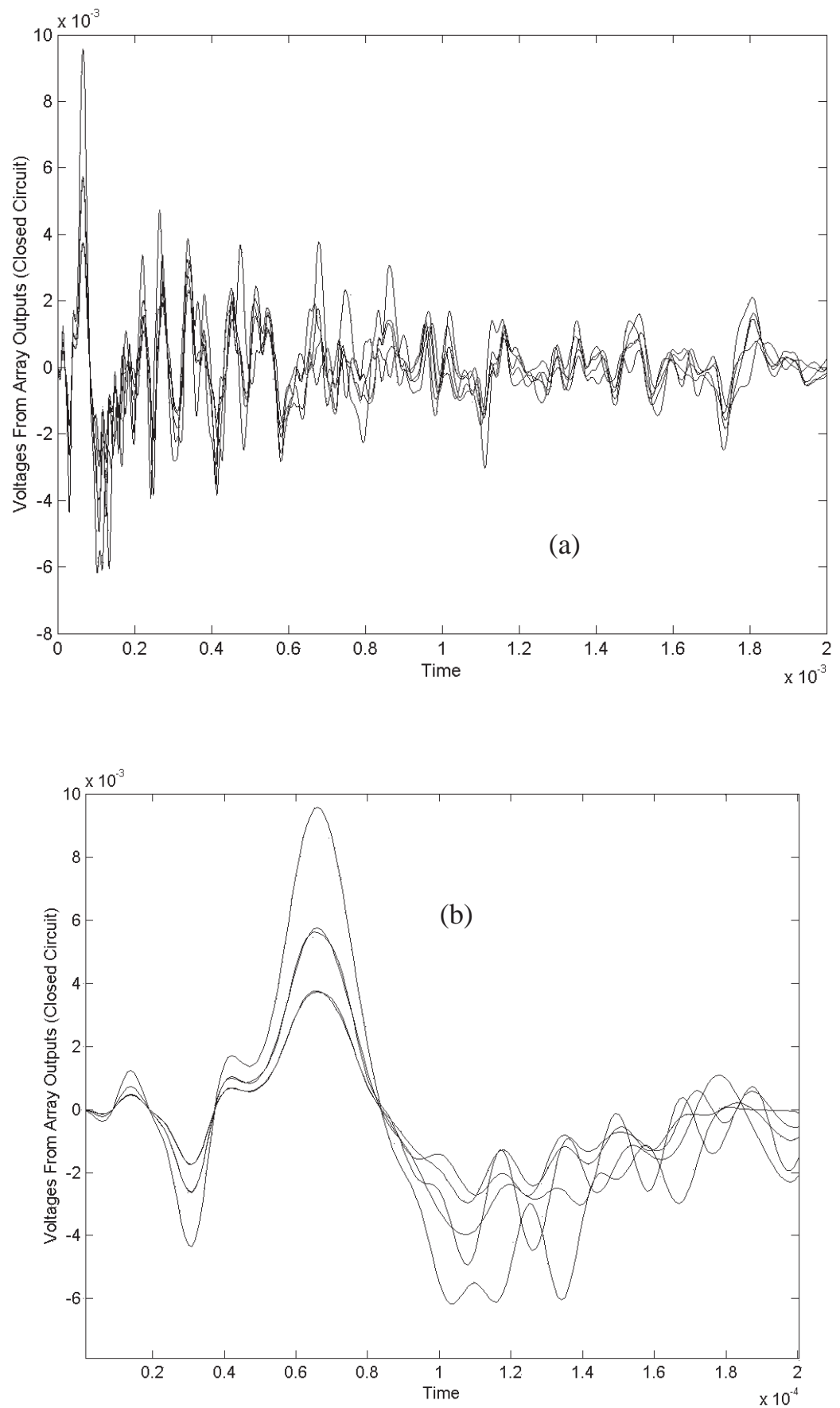

Fig. 9. Voltage time history due to edge actuation by a PZT patch; (a) array voltage for $2 \mathrm{~ms}$, (b) array voltage for $0.2 \mathrm{~ms}$.

where the subscript PZT is used to denote the strain in the PZT element. The strains in the isotropic plate are expressed as follows:

$$
\left(\varepsilon_{x}\right)_{p}=\begin{gathered}
\left(\sigma_{x}\right)_{p} \\
E_{p}-v_{y_{p}} \cdot\left(\sigma_{y}\right)_{p} \\
E_{p}
\end{gathered}
$$



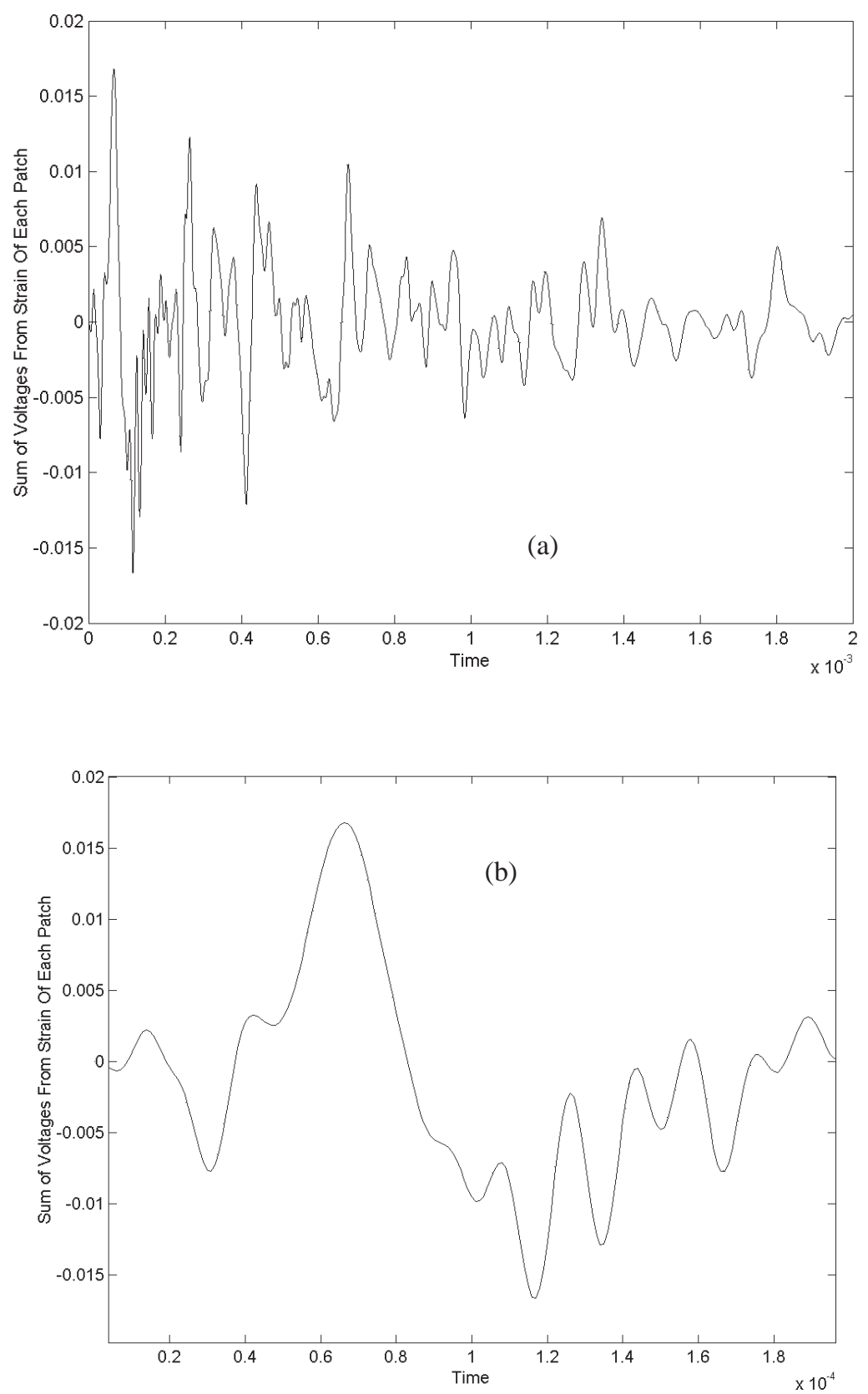

Fig. 10. Voltage time history due to edge actuation by a PZT patch; (a) continuous sensor voltage for 2 ms, (b) continuous sensor voltage for $0.2 \mathrm{~ms}$.

$$
\left(\varepsilon_{y}\right)_{p}=\begin{gathered}
\left(\sigma_{y}\right)_{p} \\
E_{p}-v_{x_{p}} \cdot\left(\sigma_{x}\right)_{p} \\
E_{p}
\end{gathered}
$$

where the subscript $p$ denotes strains in the plate [28,29]. From the equations for plate strain (Eqs (19) and (20)), the normal stresses can now be derived. Since the plate is isotropic, Poisson's ratio is assumed to be the same in the xand y-direction, thus $\nu_{y}$ and $\nu_{x}$ of the plate can be represented by $\nu_{p}$. The normal plate stresses are shown in Fig. 1 and are written as: 


$$
\begin{aligned}
& \left(\sigma_{x}\right)_{p}=\left(\begin{array}{c}
E_{p} \\
1-\nu_{p}^{2}
\end{array}\right) \cdot\left(\left(\varepsilon_{x}\right)_{p}+\nu_{p} \cdot\left(\varepsilon_{y}\right)_{p}\right) \\
& \left(\sigma_{y}\right)_{p}=\left(\begin{array}{c}
E_{p} \\
1-\nu_{p}^{2}
\end{array}\right) \cdot\left(\left(\varepsilon_{y}\right)_{p}+\nu_{p} \cdot\left(\varepsilon_{x}\right)_{p}\right)
\end{aligned}
$$

The normal stresses distributed over the lateral sides of the element can be reduced to couples per unit length, expressed as:

$$
\begin{aligned}
& m_{x}^{1} d y=\int_{-h}^{h}{ }_{-h}^{h}\left(\sigma_{x}\right)_{p} z d y d z \\
& m_{y}^{1} d x=\int_{-\frac{h}{h}}^{2}\left(\sigma_{y}\right)_{p} z d x d z
\end{aligned}
$$

where $m_{x}$ and $m_{y}$ are the moments at the plate-PZT interface per unit length. From the assumption of pure bending, the plate curvatures ${ }_{r_{x}}^{1}$ and ${ }_{r_{y}}^{1}$ can be related to the strains as follows:

$$
\begin{aligned}
& \left(\varepsilon_{x}\right)_{p}=\begin{array}{c}
z \\
r_{x}
\end{array} \\
& \left(\varepsilon_{y}\right)_{p}=\begin{array}{c}
z \\
r_{y}
\end{array}
\end{aligned}
$$

Substituting Eq. (21) into Eq. (22) yields:

$$
\begin{aligned}
& m_{x}^{1} d y=\int_{-\frac{h}{2}}^{\substack{h \\
2}}\left(\begin{array}{c}
E_{p} \\
1-\nu_{p}^{2}
\end{array}\right) \cdot\left(\left(\varepsilon_{x}\right)_{p}+\nu_{p} \cdot\left(\varepsilon_{y}\right)_{p}\right) z d y d z \\
& m_{y}^{1} d x=\int_{-\frac{h}{2}}^{\substack{h \\
2}}\left(\begin{array}{c}
E_{p} \\
1-\nu_{p}^{2}
\end{array}\right) \cdot\left(\left(\varepsilon_{y}\right)_{p}+\nu_{p} \cdot\left(\varepsilon_{x}\right)_{p}\right) z d x d z
\end{aligned}
$$

By substituting Eq. (23) into Eq. (24), the moments per unit length can be expressed by the plate curvatures, as:

$$
\begin{aligned}
& m_{x}^{1} d y=\left(\begin{array}{c}
E_{p} \\
1-\nu_{p}^{2}
\end{array}\right) \cdot \int_{-\frac{h}{2}}^{\substack{2 \\
r_{x_{p}}}}\left(\begin{array}{c}
1 \\
\nu_{y_{p}}
\end{array}\right) z^{2} d y d z \\
& m_{y}^{1} d x=\left(\begin{array}{c}
E_{p} \\
1-\nu_{p}^{2}
\end{array}\right) \cdot \int_{-\frac{h}{2}}^{\substack{h \\
r_{y_{p}}}}\left(\begin{array}{c}
1 \\
r_{x_{p}}
\end{array}\right) z^{2} d x d z
\end{aligned}
$$

Simplification results in the following form:

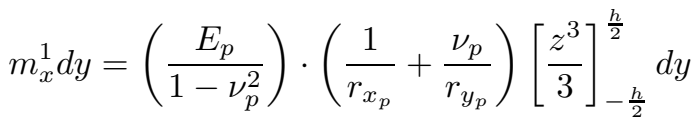

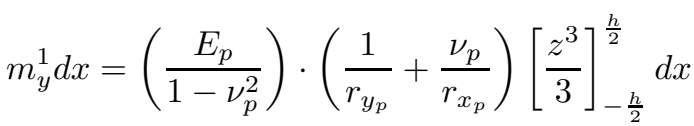

Furthermore,

$$
\begin{aligned}
& m_{x}^{1}=\left(\begin{array}{c}
E_{p} \\
1-\nu_{p}^{2}
\end{array}\right) \cdot\left(\begin{array}{c}
1 \\
r_{x_{p}}
\end{array} \begin{array}{c}
\nu_{p} \\
r_{y_{p}}
\end{array}\right)\left[\begin{array}{c}
h^{3} \\
12
\end{array}\right] \\
& m_{y}^{1}=\left(\begin{array}{c}
E_{p} \\
1-\nu_{p}^{2}
\end{array}\right) \cdot\left(\begin{array}{c}
1 \\
r_{y_{p}}
\end{array}+\begin{array}{c}
\nu_{p} \\
r_{x_{p}}
\end{array}\right)\left[\begin{array}{l}
h^{3} \\
12
\end{array}\right]
\end{aligned}
$$



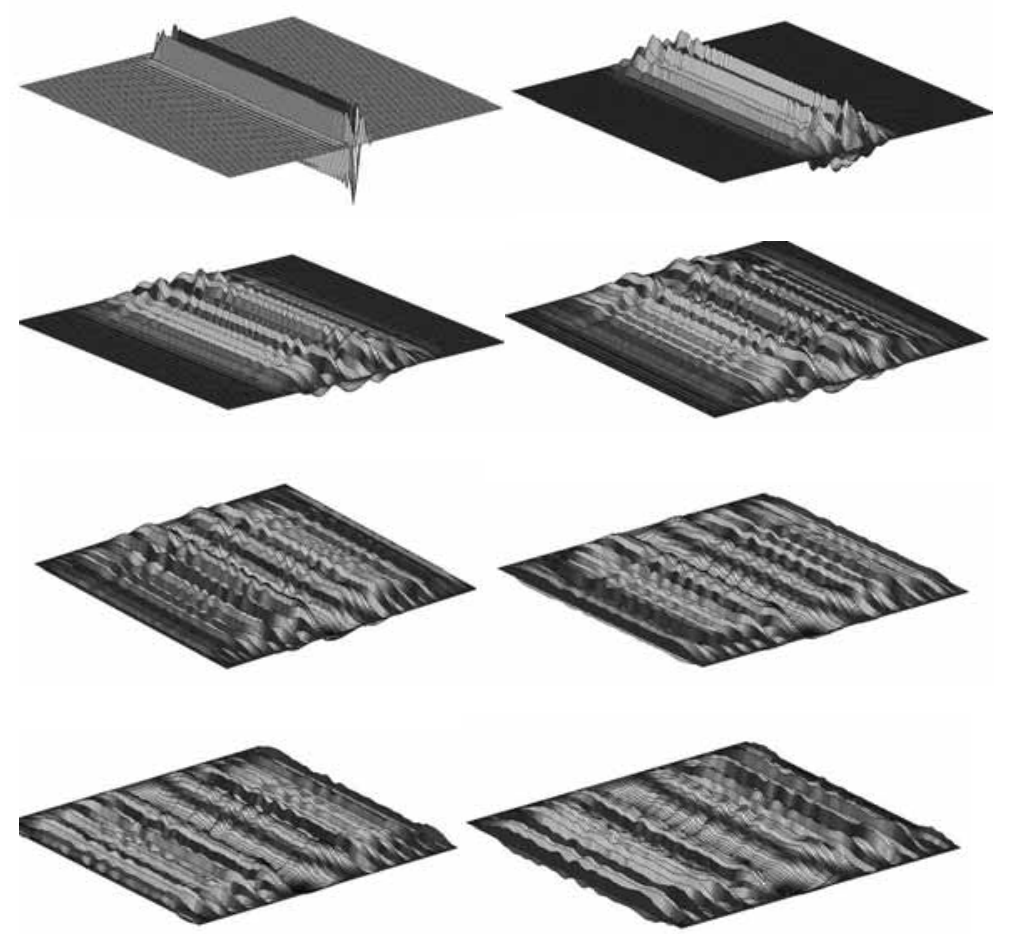

Fig. 11. Wave propagation at 10, 60, 110, 160, 210, 260, 310, 360 micro-sec due to center actuation by a wide PZT patch.

The flexural rigidity of the plate is defined as $D=\begin{gathered}E_{p} \cdot h^{3} \\ 12\left(1-\nu_{p}^{2}\right)\end{gathered}$. Equation (27) can be written as:

$$
\begin{aligned}
& m_{x}^{1}=D \cdot\left(\begin{array}{c}
1 \\
r_{x_{p}}
\end{array} \begin{array}{c}
\nu_{p} \\
r_{y_{p}}
\end{array}\right) \\
& m_{y}^{1}=D \cdot\left(\begin{array}{c}
1 \\
r_{y_{p}}
\end{array}+\begin{array}{c}
\nu_{p} \\
r_{x_{p}}
\end{array}\right)
\end{aligned}
$$

Unlike References [23,24] the moments per unit length $\left(m_{x}\right.$ and $\left.m_{y}\right)$, are not assumed to be equal in the present case. Through algebraic manipulation of Eq. (28), the plate curvatures can be expressed by the moments per unit length and the plate rigidity as follows:

$$
\begin{aligned}
& r_{x_{p}}^{1}=\frac{1}{D} \cdot\left(\begin{array}{c}
m_{x}^{1}-\nu_{p} m_{y}^{1} \\
1-\nu_{p}^{2}
\end{array}\right) \\
& r_{y_{p}}^{1}=\frac{1}{D} \cdot\left(\begin{array}{c}
m_{y}^{1}-\nu_{p} m_{x}^{1} \\
1-\nu_{p}^{2}
\end{array}\right)
\end{aligned}
$$

These results will be used at a later stage in this derivation. Now the stress-strain relationship at the interface of the plate and PZT can be derived using Eq. (23), and written as:

$$
\begin{aligned}
& \left(\sigma_{x_{i}}\right)_{p}=\left(\begin{array}{c}
E_{p} \\
1-\nu_{p}^{2}
\end{array}\right) \cdot\left(\left(\varepsilon_{x_{i}}\right)_{p}+\nu_{p} \cdot\left(\varepsilon_{y_{i}}\right)_{p}\right) \\
& \left(\sigma_{y_{i}}\right)_{p}=\left(\begin{array}{c}
E_{p} \\
1-\nu_{p}^{2}
\end{array}\right) \cdot\left(\left(\varepsilon_{y_{i}}\right)_{p}+\nu_{p} \cdot\left(\varepsilon_{x_{i}}\right)_{p}\right)
\end{aligned}
$$

The stresses at the interface of the actuator and the plate are calculated using the superposition of the external plate strains at the interface and the unconstrained PZT element strains. This gives: 

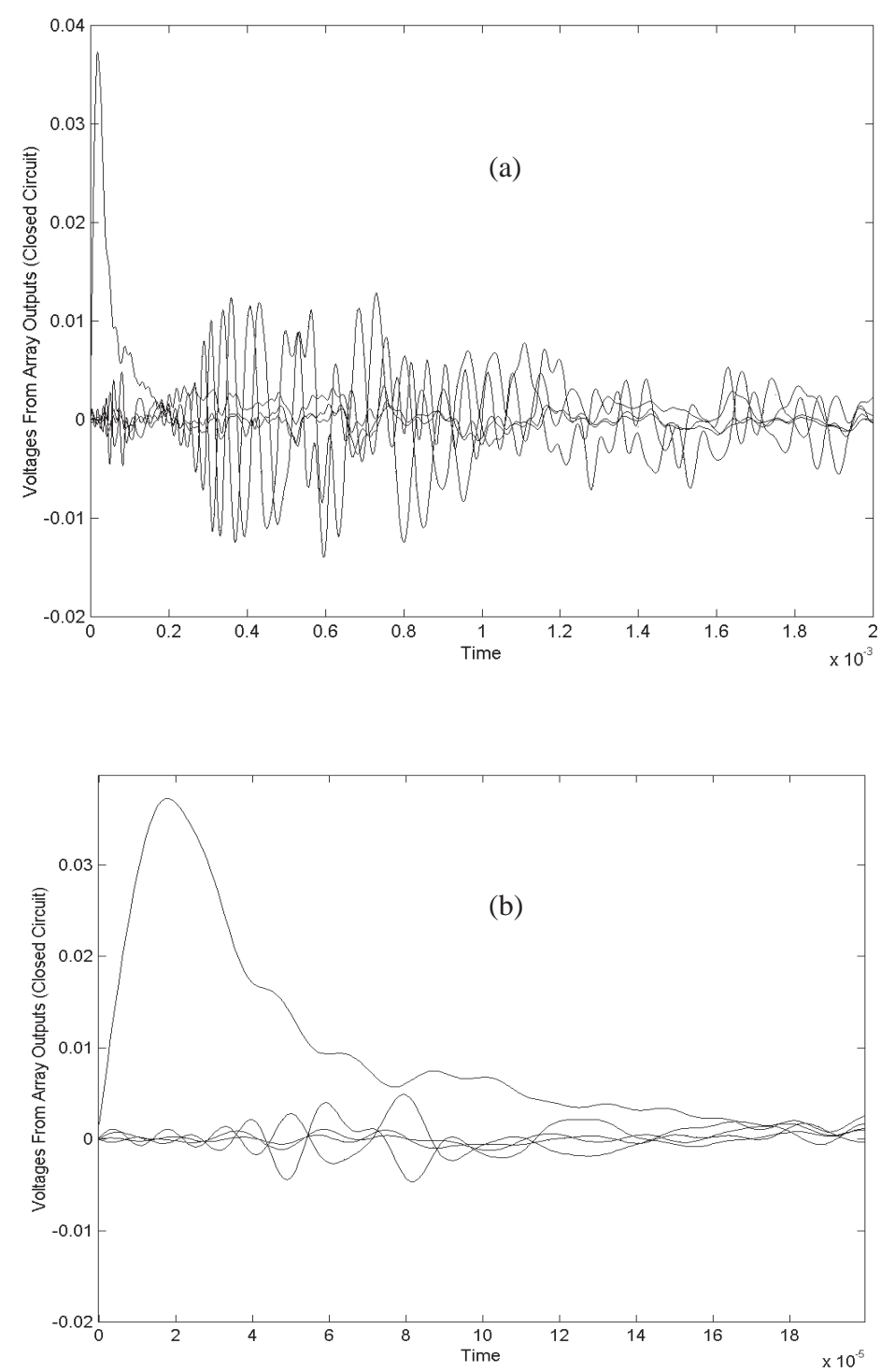

Fig. 12. Voltage time history due to center actuation by a wide PZT patch; (a) array voltage for 2 ms, (b) array voltage for 0.2 ms.

$$
\begin{aligned}
& \left(\sigma_{x_{i}}\right)_{p z t}=\left(\begin{array}{c}
E_{p z t} \\
1-\nu_{p z t}^{2}
\end{array}\right) \cdot\left(\left(\varepsilon_{x_{i}}\right)_{p z t}+\nu_{p z t} \cdot\left(\varepsilon_{y_{i}}\right)_{p z t}-\left(\varepsilon_{x}\right)_{p z t}-\nu_{p z t} \cdot\left(\varepsilon_{y}\right)_{p z t}\right) \\
& \left(\sigma_{y_{i}}\right)_{p z t}=\left(\begin{array}{c}
E_{p z t} \\
1-\nu_{p z t}^{2}
\end{array}\right) \cdot\left(\left(\varepsilon_{y_{i}}\right)_{p z t}+\nu_{p z t} \cdot\left(\varepsilon_{x_{i}}\right)_{p z t}-\left(\varepsilon_{y}\right)_{p z t}-\nu_{p z t} \cdot\left(\varepsilon_{x}\right)_{p z t}\right)
\end{aligned}
$$

The bending stresses distribution in the plate is linear through the thickness of the plate, with respect to $z$ and can be written in terms of the interface stresses giving:

$$
\sigma_{x_{p}}=\begin{gathered}
\left(\sigma_{x_{i}}\right)_{p} z \\
h \\
2
\end{gathered}, \sigma_{y_{p}}=\begin{gathered}
\left(\sigma_{y_{i}}\right)_{p} z \\
h \\
2
\end{gathered}
$$

Since the strain distribution in the PZT element is assumed to have the same slope as the strain distribution in the plate, the PZT element stresses can be written as follows: 

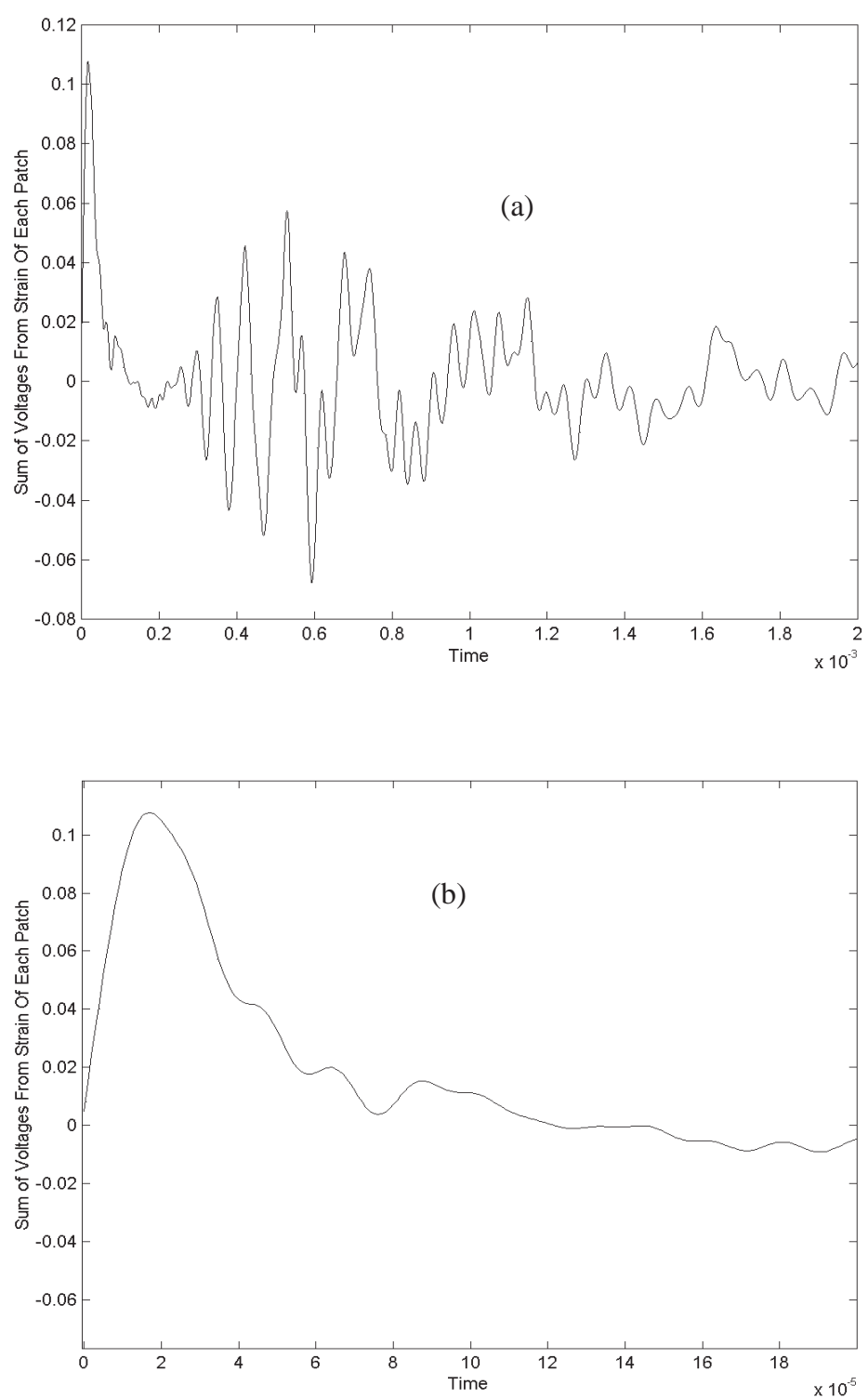

Fig. 13. Voltage time history due to center actuation by a wide PZT patch; (a) continuous sensor voltage for $2 \mathrm{~ms}$, (b) continuous sensor voltage for $0.2 \mathrm{~ms}$.

$$
\begin{gathered}
\sigma_{x_{p z t}}=\left(\sigma_{x_{i}}\right)_{p z t}-\left(\sigma_{x_{i}}\right)_{p} \cdot\left(1-\begin{array}{c}
z \\
(h / 2)
\end{array}\right) \\
\sigma_{y_{p z t}}=\left(\sigma_{y_{i}}\right)_{p z t}-\left(\sigma_{y_{i}}\right)_{p} \cdot\left(1-\begin{array}{c}
z \\
(h / 2)
\end{array}\right)
\end{gathered}
$$

The derivations for $\sigma_{p z t}$ in the $x-$ and $y$-directions are similar due of the assumption of pure bending. Only the derivation for the $x$-direction will be presented for brevity. The interface strains and the plate bending stresses must be derived in terms of the constituent material properties and the unconstrained actuator strains. The relationship is determined from a moment balance at the interface based upon the assumption that the plate stresses are due to the PZT element acting upon it. The relationship is given as: 


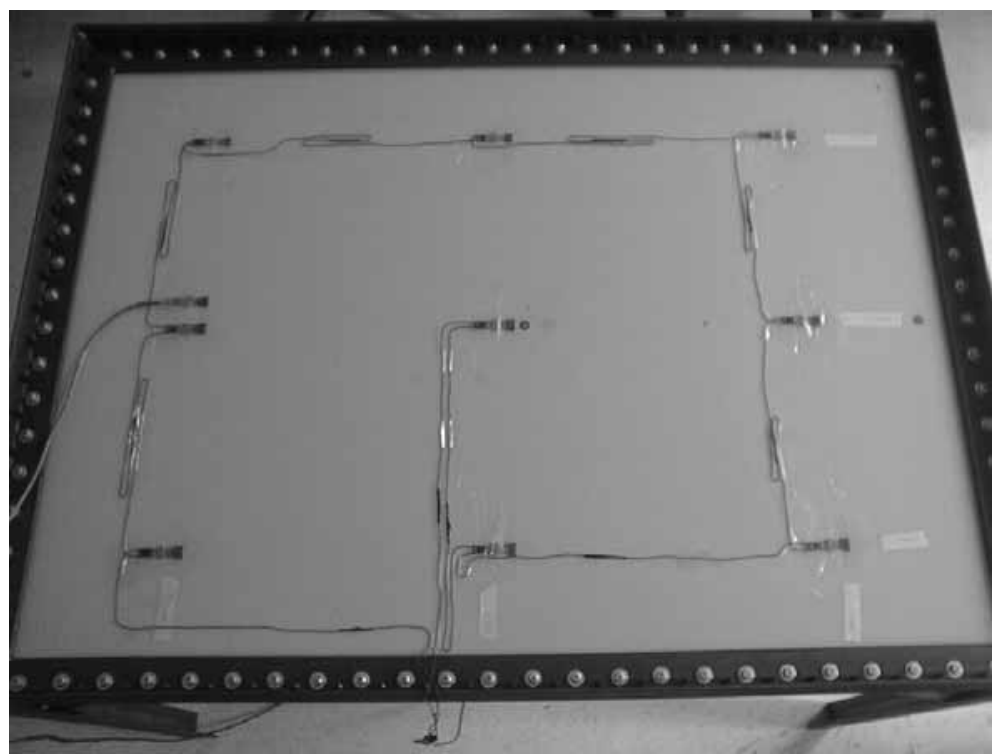

Fig. 14. Fiberglass panel with a Continuous Sensor.

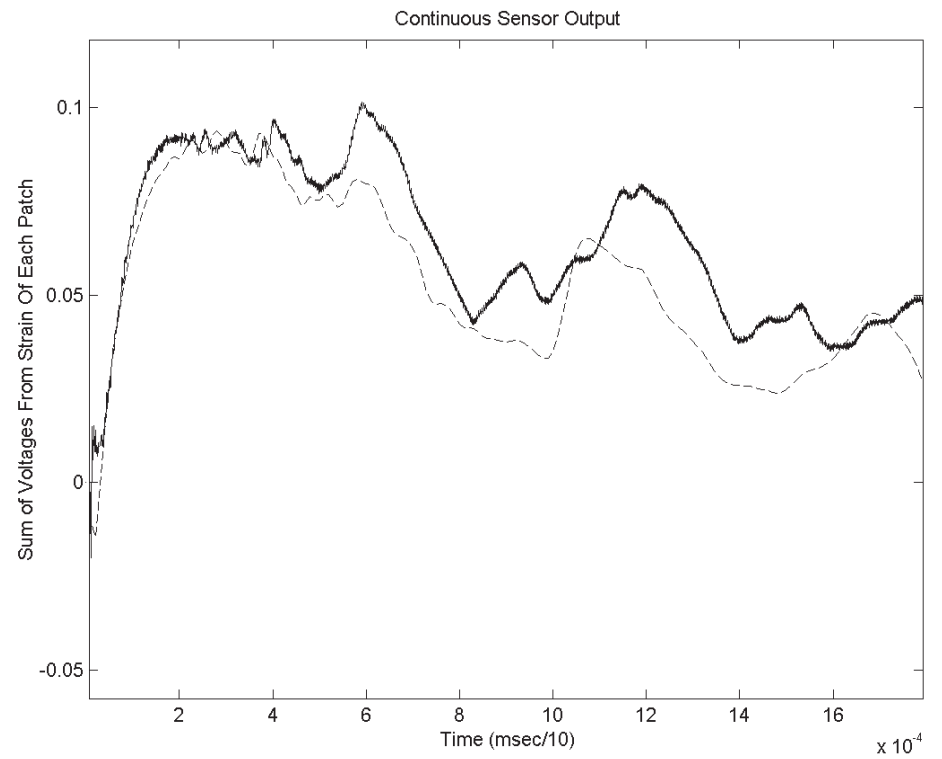

Fig. 15. Continuous Sensor response due to a lead break (simulation scaled - dashed, test - solid line).

$$
\int_{0}^{\frac{h}{2}}\left(\sigma_{x}\right)_{p} z d z+\int_{\substack{h \\ 2}}^{\frac{h}{2}+t_{p z t}}\left(\sigma_{x}\right)_{p z t} z d z=0
$$

Substitution of Eqs (32) and (33) into Eq. (34) yields:

$$
\int_{0}^{h} \begin{gathered}
h \\
2 \\
\left(\sigma_{x_{i}}\right)_{p}
\end{gathered}+\int_{\substack{h \\
2}}^{\substack{h \\
2_{2}}}\left(\left(\sigma_{x_{i}}\right)_{p z t}-\left(\sigma_{x_{i}}\right)_{p}+\left(\sigma_{x_{i}}\right)_{p} \begin{array}{c}
z \\
h / 2
\end{array}\right) z d z=0
$$




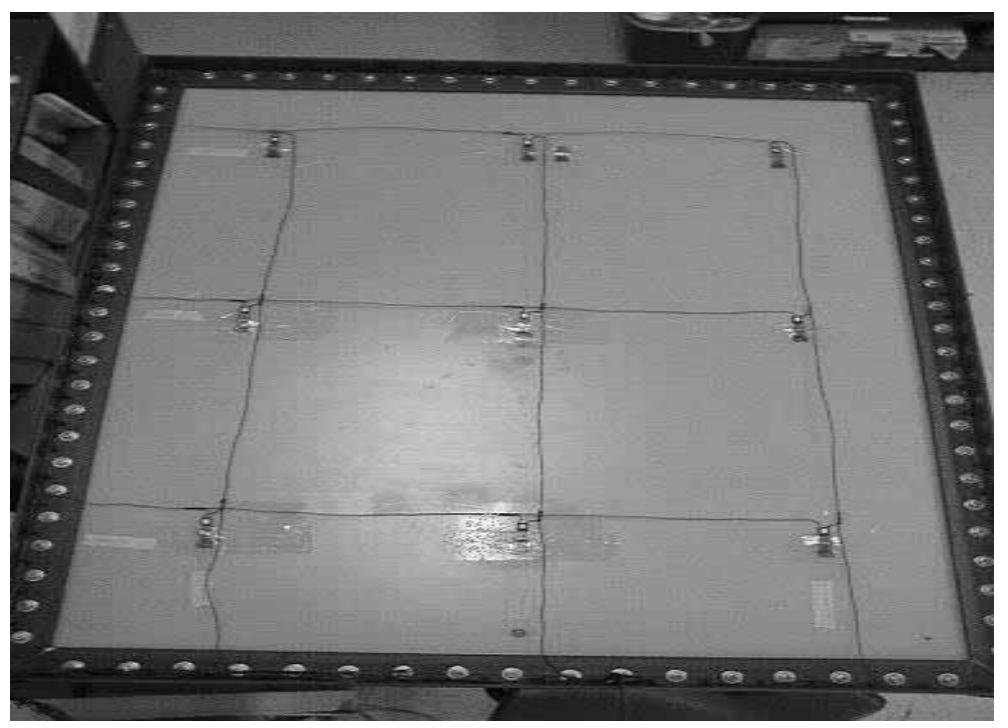

Fig. 16. Fiberglass panel with Cross Array Sensor Architecture.

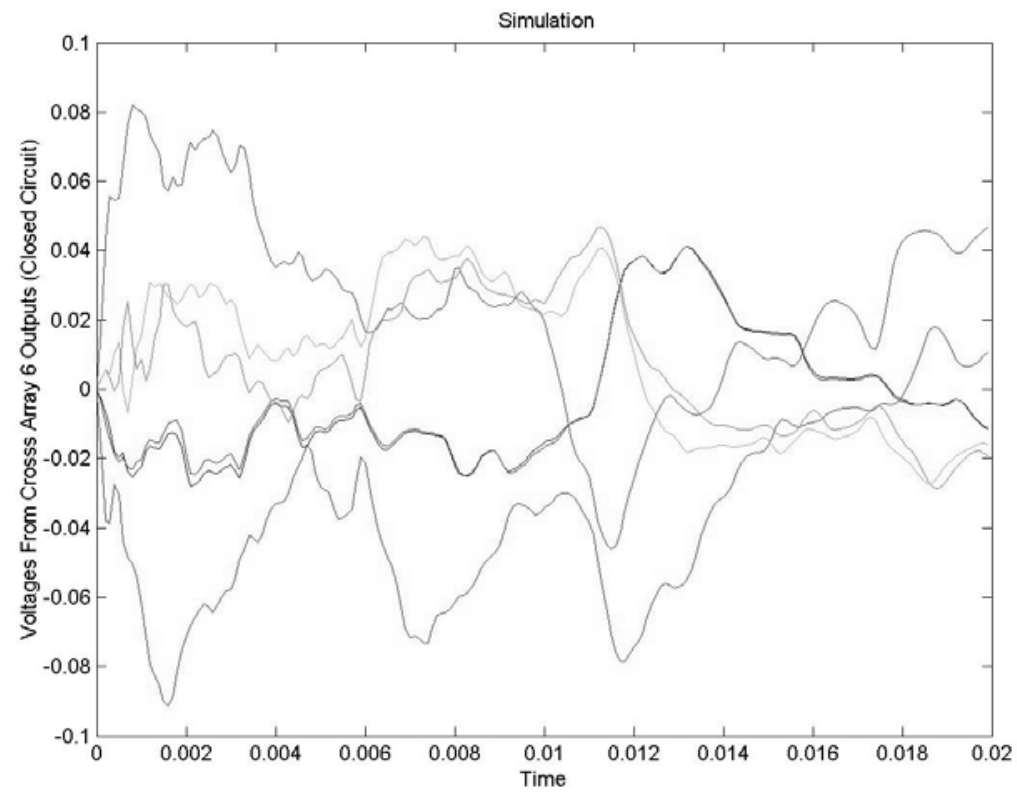

Fig. 17. Simulation scaled response of the Cross. Array (6 outputs), center excitation.

Evaluation of the integrals yields:

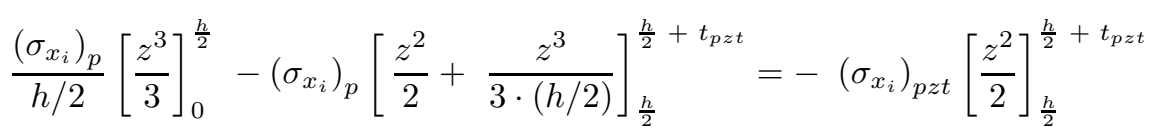

From Eq. (36) the relationship between $\left(\sigma_{x i}\right)_{p}$ and $\left(\sigma_{x i}\right)_{p z t}$ is given as:

$$
K=\frac{\left(\sigma_{x_{i}}\right)_{p}}{\left(\sigma_{x_{i}}\right)_{p z t}}=-\left(\begin{array}{c}
t^{2}+t h \\
t^{2}+\left(h^{2} / 6\right)+\left(4 t^{3} / 3 h\right)
\end{array}\right)
$$




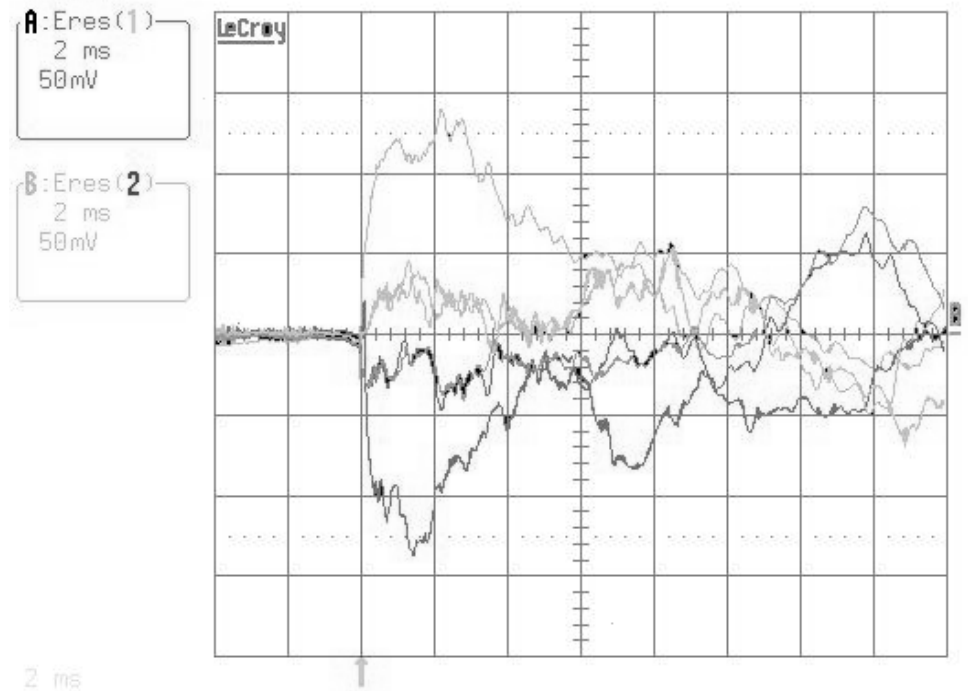

Fig. 18. Test response of the actual Cross Array with all outputs, center excitation.

Similarly,

$$
K=\frac{\left(\sigma_{y_{i}}\right)_{p}}{\left(\sigma_{y_{i}}\right)_{p z t}}=-\left(\begin{array}{c}
t^{2}+t h \\
t^{2}+\left(h^{2} / 6\right)+\left(4 t^{3} / 3 h\right)
\end{array}\right)
$$

Now, substituting Eqs (30) and (31) into Eq. (38) gives the following:

$$
\begin{gathered}
1 \\
K
\end{gathered}=\begin{gathered}
\left(\sigma_{x_{i}}\right)_{p z t} \\
\left(\sigma_{x_{i}}\right)_{p}
\end{gathered}=\begin{gathered}
\left(\begin{array}{c}
E_{p z t} \\
1-\nu_{p z t}^{2}
\end{array}\right) \cdot\left(\left(\varepsilon_{x_{i}}\right)_{p z t}+\nu_{p z t} \cdot\left(\varepsilon_{y_{i}}\right)_{p z t}-\left(\varepsilon_{x}\right)_{p z t}-\nu_{p z t} \cdot\left(\varepsilon_{y}\right)_{p z t}\right) \\
\left(\begin{array}{c}
E_{p} \\
1-\nu_{p}^{2}
\end{array}\right) \cdot\left(\left(\varepsilon_{x_{i}}\right)_{p}+\nu_{p} \cdot\left(\varepsilon_{y_{i}}\right)_{p}\right)
\end{gathered}
$$

Defining a variable $P$ as follows:

$$
P=\frac{\left(\begin{array}{c}
E_{p z t} \\
1-\nu_{p z t}^{2}
\end{array}\right)}{\left(\begin{array}{c}
E_{p} \\
1-\nu_{p}^{2}
\end{array}\right)} K,
$$

Multiplying Eq. (39) by $K$, and substituting $P$, the following expression is obtained:

$$
\begin{gathered}
1=P \cdot \begin{array}{c}
\left(\left(\varepsilon_{x_{i}}\right)_{p z t}+\nu_{p z t} \cdot\left(\varepsilon_{y_{i}}\right)_{p z t}-\left(\varepsilon_{x}\right)_{p z t}-\nu_{p z t} \cdot\left(\varepsilon_{y}\right)_{p z t}\right) \\
\left(\left(\varepsilon_{x_{i}}\right)_{p}+\nu_{p} \cdot\left(\varepsilon_{y_{i}}\right)_{p}\right)
\end{array} \\
\begin{array}{c}
P \cdot\left(\left(\varepsilon_{x_{i}}\right)_{p z t}+\nu_{p z t} \cdot\left(\varepsilon_{y_{i}}\right)_{p z t}\right)-P \cdot\left(\left(\varepsilon_{x}\right)_{p z t}-\nu_{p z t} \cdot\left(\varepsilon_{y}\right)_{p z t}\right) \\
\left(\left(\varepsilon_{x_{i}}\right)_{p}+\nu_{p} \cdot\left(\varepsilon_{y_{i}}\right)_{p}\right)
\end{array} \\
\left(\begin{array}{c}
\left.\left(\varepsilon_{x_{i}}\right)_{p}+\nu_{p} \cdot\left(\varepsilon_{y_{i}}\right)_{p}\right)=P \cdot\left(\left(\varepsilon_{x_{i}}\right)_{p z t}+\nu_{p z t} \cdot\left(\varepsilon_{y_{i}}\right)_{p z t}\right)-P \cdot\left(\left(\varepsilon_{x}\right)_{p z t}-\nu_{p z t} \cdot\left(\varepsilon_{y}\right)_{p z t}\right) \\
(1-P) \cdot\left(\varepsilon_{x_{i}}\right)_{p}+\left(\nu_{p}-\nu_{p z t} \cdot P\right) \cdot\left(\varepsilon_{y_{i}}\right)_{p}=-P \cdot\left(\varepsilon_{x}\right)_{p z t}-P \cdot \nu_{p z t} \cdot\left(\varepsilon_{y}\right)_{p z t}
\end{array}\right.
\end{gathered}
$$

Similarly, for the $y$-direction: 
Table 1

Lateral sides of an element of the PZT with the plate underneath

$\begin{array}{ccc}\begin{array}{c}\text { Sensor } \\ \text { S (row,col) }\end{array} & \begin{array}{c}\text { X-Location } \\ \text { inch }(\mathrm{m})\end{array} & \begin{array}{c}\text { Y-Location } \\ \text { inch }(\mathrm{m})\end{array} \\ \text { S11 } & 5.25(0.127) & 7.25(0.184) \\ \text { S12 } & 17.25(0.438) & 7.25(0.184) \\ \text { S13 } & 29.25(0.743) & 7.25(0.184) \\ \text { S21 } & 5.25(0.127) & 23.875(0.61) \\ \text { S22 } & 17.25(0.438) & 23.875(0.61) \\ \text { S23 } & 29.25(0.743) & 23.875(0.61) \\ \text { S31 } & 5.25(0.127) & 40.5(1.03) \\ \text { S32 } & 17.25(0.438) & 40.5(1.03) \\ \text { S33 } & 29.25(0.743) & 40.5(1.03)\end{array}$

$$
(1-P) \cdot\left(\varepsilon_{y_{i}}\right)_{p}+\left(\nu_{p}-\nu_{p z t} \cdot P\right) \cdot\left(\varepsilon_{x_{i}}\right)_{p}=-P \cdot\left(\varepsilon_{y}\right)_{p z t}-P \cdot \nu_{p z t} \cdot\left(\varepsilon_{x}\right)_{p z t}
$$

In matrix form, Eqs (44) and (45) can be written as follows:

$$
\begin{aligned}
& {\left[\begin{array}{lc}
(1-P) & \left(\nu_{p}-\nu_{p z t} \cdot P\right) \\
\left(\nu_{p}-\nu_{p z t} \cdot P\right) & (1-P)
\end{array}\right] \cdot\left[\begin{array}{l}
\varepsilon_{x_{i_{p}}} \\
\varepsilon_{y_{i_{p}}}
\end{array}\right]=-P \cdot\left[\begin{array}{ll}
1 & \nu_{p z t} \\
\nu_{p z t} & 1
\end{array}\right] \cdot\left[\begin{array}{l}
\varepsilon_{x_{p z t}} \\
\varepsilon_{y_{p z t}}
\end{array}\right]} \\
& {\left[\begin{array}{l}
\varepsilon_{x_{i_{p}}} \\
\varepsilon_{y_{i_{p}}}
\end{array}\right]=-P \cdot\left[\begin{array}{lr}
(1-P) & \left(\nu_{p}-\nu_{p z t} \cdot P\right) \\
\left(\nu_{p}-\nu_{p z t} \cdot P\right) & (1-P)
\end{array}\right]^{-1} \cdot\left[\begin{array}{ll}
1 & \nu_{p z t} \\
\nu_{p z t} & 1
\end{array}\right] \cdot\left[\begin{array}{l}
\varepsilon_{x_{p z t}} \\
\varepsilon_{y_{p z t}}
\end{array}\right]}
\end{aligned}
$$

Evaluation of the matrix inverse in Eq. (46) yields:

$$
\left[\begin{array}{l}
\varepsilon_{x_{i_{p}}} \\
\varepsilon_{y_{i_{p}}}
\end{array}\right]={ }_{(1-P)^{2}-\left(\nu_{p}-\nu_{p z t} \cdot P\right)^{2}} \cdot\left[\begin{array}{lr}
(1-P) & -\left(\nu_{p}-\nu_{p z t} \cdot P\right) \\
-\left(\nu_{p}-\nu_{p z t} \cdot P\right) & (1-P)
\end{array}\right] \cdot\left[\begin{array}{ll}
1 & \nu_{p z t} \\
\nu_{p z t} & 1
\end{array}\right] \cdot\left[\begin{array}{l}
\varepsilon_{x_{p z t}} \\
\varepsilon_{y_{p z t}}
\end{array}\right]
$$

Thus the interface strains can be reduced to a function of the unconstrained PZT element strains and the constituent material properties. The uniformly distributed edge moments per unit length that would produce the interface strains can also be determined. By substituting the result from Eq. (23) into Eq. (27) and recalling the definition of the flexural rigidity of the plate, the edge moments can be expressed as follows:

$$
\begin{aligned}
& m_{x}^{1}=\begin{array}{c}
E_{p} \cdot h^{3} \\
12\left(1-\nu_{p}^{2}\right)
\end{array} \cdot\left(\left(\varepsilon_{x}\right)_{p}+\nu_{p} \cdot\left(\varepsilon_{y}\right)_{p}\right) \begin{array}{l}
1 \\
z
\end{array} \\
& m_{y}^{1}=\begin{array}{c}
E_{p} \cdot h^{3} \\
12\left(1-\nu_{p}^{2}\right)
\end{array} \cdot\left(\left(\varepsilon_{y}\right)_{p}+\nu_{p} \cdot\left(\varepsilon_{x}\right)_{p}\right)_{z}^{1}
\end{aligned}
$$

Substituting Eqs (30) and (32) into Eq. (48) and noting that at the interface $z=h / 2$, yields:

$$
\begin{aligned}
& m_{x}^{1}=\frac{\left(\sigma_{x_{i}}\right)_{p} \cdot h^{2}}{6} \\
& m_{y}^{1}=\frac{\left(\sigma_{y_{i}}\right)_{p} \cdot h^{2}}{6}
\end{aligned}
$$

Substituting the interface stresses of the plate from Eq. (30) gives:

$$
\begin{aligned}
& m_{x}^{1}=\frac{h^{2}}{6}\left(\begin{array}{c}
E_{p} \\
1-\nu_{p}^{2}
\end{array}\right) \cdot\left(\left(\varepsilon_{x_{i}}\right)_{p}+\nu_{p} \cdot\left(\varepsilon_{y_{i}}\right)_{p}\right) \\
& m_{x}^{1}=h^{2}\left(\begin{array}{c}
E_{p} \\
1-\nu_{p}^{2}
\end{array}\right) \cdot\left(\left(\varepsilon_{y_{i}}\right)_{p}+\nu_{p} \cdot\left(\varepsilon_{x_{i}}\right)_{p}\right)
\end{aligned}
$$

In matrix form this can be written as:

$$
\left[\begin{array}{l}
m_{x}^{1} \\
m_{y}^{1}
\end{array}\right]={ }_{6}^{h^{2}}\left(\begin{array}{c}
E_{p} \\
1-\nu_{p}^{2}
\end{array}\right) \cdot\left[\begin{array}{ll}
1 & \nu_{p} \\
\nu_{p} & 1
\end{array}\right] \cdot\left[\begin{array}{l}
\varepsilon_{x_{i_{p}}} \\
\varepsilon_{y_{i_{p}}}
\end{array}\right]
$$


where the vector of the interface plate strains is given in Eq. (47). Before substituting into the plate equations, the moments produced by the PZT element are expressed as:

$$
\begin{aligned}
& m_{x}=m_{x}^{1} \cdot\left[H\left(x-x_{1}\right)-H\left(x-x_{2}\right)\right] \cdot\left[H\left(y-y_{1}\right)-H\left(y-y_{2}\right)\right] \cdot f(t) \\
& m_{y}=m_{y}^{1} \cdot\left[H\left(x-x_{1}\right)-H\left(x-x_{2}\right)\right] \cdot\left[H\left(y-y_{1}\right)-H\left(y-y_{2}\right)\right] \cdot f(t)
\end{aligned}
$$

where $f(\mathrm{t})$ is purely a function of time. When $f(\mathrm{t})$ is represented by the delta function, and the moments are used in the classical plate equations, then the results can be summarized by Eqs (16-18). Details of the derivation are presented in Appendix A. The corresponding equations for a steady state sinusoidal excitation can be similarly derived by following the same process as shown in Appendix A but with a different forcing function.

\subsection{Modeling the continuous and cross sensor array}

The advantage of using the continuous and cross sensor arrays for passive damage detection is that a large number of PZT sensor elements can be used so that one sensor will always be close to any possible damage to give a good signal to noise ratio, and only a small number of channels of data acquisition are needed. The disadvantage is that damage location is more difficult. The piezoceramic sensor nodes in the continuous and cross sensor array can be modeled as a capacitor in parallel with a current source. The piezoelectric constitutive equations are as shown in Eqs (10) and (11). The piezoceramic sensors can be modeled using the piezoelectric constitutive equations and by connecting the segments into an electric circuit as shown in Fig. 2. The output voltage equation for a continuous sensor nerve can be expressed as follows using Kirchhoff's law:

$$
\frac{d}{d t}(i)+{ }_{R C}^{n \cdot i}={ }_{R C}^{e A_{e}} \sum_{j=1}^{n} \dot{S}_{j}
$$

where $\mathrm{C}$ is the capacitance of the PZT sensor, $A_{e}$ is the effective capacitor area, $\mathrm{h}$ is the effective plate separation distance, $S$ is strain and $\dot{S}$ is the strain rate, $i_{c}$ represents the component of the current going through the capacitor of the model, and $i_{g}$ represents the component of the current generated by the piezoelectric sensor. Further details of this derivation can be found in Ref [27]. The homogeneous and particular solutions of Eq. (54) must be calculated and added to obtain the total solution for the current $i$. The product of the current $\mathrm{i}(\mathrm{t})$, and the impedance $\mathrm{R}$ of the measuring device equals the voltage of the series connected sensors as a function of time. Thus we solve for the current to get the voltage $V_{0}=i R$. This voltage is proportional to the dynamic strain in the structure at the sensor and thus can be used to detect damage through dynamic strain measurements and acoustic emissions. The current is numerically computed using the implicit Newmark-beta time marching scheme. Figure 2(a) and 2(b) shows examples of a continuous sensor array and a $3 \times 3$ cross sensor array architecture. Details of the continuous sensor and sensor array system can be found in references [27,31].

\section{Simulation of wave propagation}

The simulation is performed using a model of the glass epoxy composite plate, which is modeled as a quasiisotropic plate. Four different cases of passive and active wave generation and propagation are presented here, using a lead break excitation modeled as a step function and a surface bonded PZT actuator, and sensing using the continuous sensor and array sensor. The material properties of the glass epoxy composite plate are as follows: Elastic modulus $=29.6 \mathrm{GPa}$, density $=2200 \mathrm{~kg} / \mathrm{m}^{3}$, Poisson's ratio $=0.27$. The size of the plate is $0.88 \mathrm{~m} \times$ $1.21 \mathrm{~m} \times 3.2 \mathrm{~mm}$. The first 100 vibration modes have been used for the simulation to reduce computation time, for increased accuracy 200 or modes can be used. The damping ratio considered for all modes is 0.01 and the time step used is one microsecond. A total of $1600(40 \times 40)$ grid points are used to generate the 3-D displacement plots. It must be noted that for sensing accuracy, the length of the sensor node should be shorter than half the wavelength of the highest frequency mode that is used in the simulation. Approximate material properties of the twelve PZT sensor nodes considered in the simulation are as follows: Poisson's ratio $=0.31$; permittivity of free space (dielectric of air $)=8.854 \mathrm{e}-12\left(\right.$ Farad $/ \mathrm{m}=$ Coulomb $\left.^{\wedge} 2 / \mathrm{Nm}^{\wedge} 2\right)$. Density $=7700 \mathrm{~kg} / \mathrm{m}^{\wedge} 3$, short circuit stiffness or modulus PZT 
$\left(\mathrm{N} / \mathrm{m}^{\wedge} 2\right) \mathrm{E} 11=61.0 \mathrm{GPa}$; PZT induced strain constant $\mathrm{d}_{31}=-10.9 e-11 \mathrm{~m} / \mathrm{V}$ and $\mathrm{d}_{31}$ is assumed to be equal to $\mathrm{d}_{32}$; each of the PZT sensor node dimensions are 0.25 in X 0.5in X 0.01in $(0.00635 \mathrm{~m} \times 0.0127 \mathrm{~m} \times 0.000254 \mathrm{~m})$; PZT dielectric with respect to air is taken as 1800 . Figure 2 shows the circuit model of the continuous sensor array and the cross-sensor array, which are being currently investigated by the authors [?]27,31. Figure 3 shows the schematic view of the sensor placement on the composite plate, which is used in the present simulation. The midpoints of the sensor nodes are presented in Table 1.

Figure 4 shows the first case of propagating acoustic waves at the time snapshots 10 micro-sec to 360 microsecond due to anti-symmetric Lamb waves generated by a lead break (simulating a AE source) placed normally at the co-ordinates $(0.3048 \mathrm{~m}, 0.4064 \mathrm{~m})$ of the glass epoxy composite plate. The surface displacement profile of the plate due to the lead break excitation is depicted in this figure for the time sequences mentioned above. Figure 5 shows the active wave propagation over the times 10 microseconds to 360 microseconds due to anti-symmetric Lamb waves generated by a PZT actuator placed at the center of the glass epoxy composite plate. The actuator dimensions are $2.5 \mathrm{~cm} \times 5 \mathrm{~cm} \times 0.25 \mathrm{~mm}$. Figures 4 and 5 show the difference in wave generation and propagation due to the input excitation. Figures 6 and 7 show the voltage-time history of the cross-array and the continuous array sensors attached to the plate, respectively, caused by the center actuation by the PZT patch as shown in Fig. 5. Figure 6(a) shows the voltage array outputs for $2 \mathrm{~ms}$, whereas Fig. 6(b) zooms in to look into the leading edges of the time history of each of the outputs. Figure 7 depicts the time history for a single continuous sensor, which is comprised all of the sensor nodes as shown in Fig. 3 but connected in series to give a single output. Both sensing architectures are considered in order to minimize the number of channels of data output that are needed. For the array sensing architecture and an NXN array cluster, there would be 2N-1 Digital to Analog (DAQ) outputs with one channel acting as a ground. For the continuous sensor with $\mathrm{N}$ sensor nodes, there is only one output channel. Since multiple sensors are connected together, the acoustic signals will combine to form a complex signal as shown in Fig. 7(a). The high frequency content of the composite signal indicates that an acoustic emission has occurred. Each of the voltage outputs must be analyzed using signal processing techniques to determine the acoustic emission source locations and the waveform characteristics. The leading edges of the voltage time history outputs of the array in Fig. 6(b) show the higher amplitude of the outputs for the array rows and columns that are closer to the center actuator. Similar observations can also be made for the later examples. Figure 8 shows the anti-symmetric Lamb wave propagation for the time 10 microseconds to 360 microseconds due to the PZT actuator placed at one edge of the glass epoxy composite plate. The actuator dimensions are $2.5 \mathrm{~cm} \times 5 \mathrm{~cm} \times 0.25 \mathrm{~mm}$. In this case, the incident waves are scattered by the reflected waves because the actuator is located very close to one of the boundaries. Figures 9 and 10 show the voltage-time history of the cross-array and the continuous array sensors modeled on the plate, respectively. The differences in the voltage time history between the two cases (Figs 6-7 and 9-10) can be studied to determine the optimal placement of the actuator considering scattering of the waves. Figure 11 shows the active wave propagation at the time 10 micro-sec to 360 micro-sec due to anti-symmetric Lamb waves generated by a PZT actuator placed at the center and across the width of the glass epoxy composite plate. The actuator dimensions are $0.81 \mathrm{~m} \times 2.5 \mathrm{~cm} \times 0.25 \mathrm{~mm}$. Figures 12 and 13 show the voltage-time histories of the cross-array and continuous array sensors, respectively. The animated displacements of the plate show the wave generation, propagation, and scattering of the incident wavefront due to the collision with the reflected waves, and the corresponding effect on the voltage outputs. Figures 5 and 8 show that the small rectangular discrete PZT actuator will generate circular wave fronts, while Fig. 11 shows that a long actuator across the width of the plate can generate a shock front type wave, which may have interesting applications. In all of the cases, the PZT voltage outputs are high enough to be detected without amplification.

Some preliminary results validating the models of the continuous sensor and sensor array system are presented here. Figure 14 shows a fiberglass panel with a continuous sensor with panel dimensions specified in the previous example. The sensor node locations on the panel are given in Table 1, the sensor connections are shown in Fig. 3. Figure 15 shows the response of the continuous sensor due to a lead break on the panel. The simulation result is represented by the scaled dashed line; the test result is represented by the solid line. The simulation result was scaled in amplitude to show the comparison of waveform types. The experimental result would be closer in amplitude to the simulation if the exact sensor capacitance and cable resistance properties were measured and used in the simulation. Figure 16 shows the fiberglass panel with the cross array sensor architecture. Simulation responses of the cross array sensory outputs due to center excitation is shown in Fig. 17. Figure 18 shows the experimental responses of the Cross Array sensor architecture (as depicted in Fig. 16) with all outputs due to a center excitation. The reasonable 
agreement of the simulated and experimental results indicates the usefulness of the modeling of the plate and the sensor systems. Detailed experimental verification and testing of the above models using the voltage history outputs is shown in $[27,31]$.

This is the only closed-form model of asymmetric wave propagation with dispersion, damping, and reflection in a plate that the authors are aware of. The model is a useful tool for the design of sensor and actuator systems for SHM. The model is reasonably accurate for thin plates. For thicker plates, the plate frequencies and wave speeds will contain some error, but the wave generation, propagation, and reflection characteristics can still be simulated and studied. A comparison of phase velocities using this model and Lamb wave theory for a composite plate is given in reference [32]. Further investigations are being done to correlate the peaks in the voltage time history graphs with the time when the incident and the reflected waves hit the sensors, and when the waves are scattered from a crack embedded in the plate. A neural network algorithm has been devised as one possible approach to detect and locate the crack in the plate model [27,31]. Other signal processing techniques are being studied to locate the source of the AE signal using perpendicular continuous sensor arrangement [33]. The near real-time interpretation of the damage signals from continuous and cross-sensor arrays is an area of continuing development. An approach of using rectangular arrays of continuous sensor neurons is discussed in [32,34].

\section{Conclusions}

The simulations illustrate how high frequency flexural acoustic waves are generated by simulated AE sources, and by PZT patch actuators. This demonstrates both passive and active wave propagation. The incident wavefronts and the scattering due to the reflected waves are clearly shown in the simulations. The physics based model thus developed can be a helpful tool for structural health monitoring scientists and engineers to study the acoustic wave generation and propagation in plates. The simulations performed have shown that multiple piezoelectric patches can be connected together in a series or array pattern to simulate the way biological nerves have multiple inputs (dendrites) connected together. This reduces the number of channels of data acquisition needed to detect damage represented by acoustic emissions or high strains. The simulation model can also be used to optimize the design of the neural system for different structural materials and sizes. This can also be used for simulating active interrogation for damage detection in plate type structures. The model includes damping and dispersion and is helpful to determine the actuator and sensor spacing and locations for both the continuous sensor and for the sensor array configurations. The model can also be used for devising a neural network analysis for detection, localization, and quantification of the crack/damage and monitoring the survivability of the plate structure. The continuous and cross-sensor array allow a large number of sensors to be used for passive detection of propagating damage using a small number of channels of data acquisition.

\section{Acknowledgement}

Support of this research by the National Research Council, the Nondestructive Evaluation Sciences Branch (NESB) of NASA Langley Research Center, the National Renewable Energy Laboratory, and the Smart Structures Laboratory of Arizona State University is gratefully acknowledged.

Appendix A - Derivation of impulse input excitation to a PZT patch on a simply supported plate

The activated piezoelectric actuator will induce internal moments across the PZT. The impulse moments in the plate can be written using the Heaviside step function as:

$$
\begin{aligned}
& m_{x}=m_{x}^{1} \cdot\left[H\left(x-x_{1}\right)-H\left(x-x_{2}\right)\right] \cdot\left[H\left(y-y_{1}\right)-H\left(y-y_{2}\right)\right] \cdot \delta(t) \\
& m_{y}=m_{y}^{1} \cdot\left[H\left(x-x_{1}\right)-H\left(x-x_{2}\right)\right] \cdot\left[H\left(y-y_{1}\right)-H\left(y-y_{2}\right)\right] \cdot \delta(t)
\end{aligned}
$$

From the classical theory of plates and vibration of plates, the equation of motion can be written in terms of internal plate flexural moments $M_{x}, M_{y}$ and $M_{x y}$ and actuator induced moments $m_{x}$ and $m_{y}$ and is: 


$$
\underset{\partial x^{2}}{\partial^{2}}\left(M_{x}-m_{x}\right)+2 \frac{\partial^{2} M_{x y}}{\partial x \partial y}+\frac{\partial^{2}}{\partial y^{2}}\left(M_{y}-m_{y}\right)+\rho h \ddot{w}=0
$$

We can express Eq. (A2) in terms of displacement as [28]:

$$
D \nabla^{4} w(x, y, t)+\rho h \ddot{w}=\begin{gathered}
\partial^{2} m_{x} \\
\partial x^{2}
\end{gathered}+\begin{gathered}
\partial^{2} m_{y} \\
\partial y^{2}
\end{gathered}
$$

The boundary conditions for the simply supported plate are given by Eqs (2) and (3). The temporal equation is derived in terms of modes using separation of spatial and time variables, and orthogonal relationships of trigonometric functions. A similar procedure was followed in the lead break analysis to solve for the plate displacements due to Hsu-Nielsen source excitation. The plate displacement is expressed in terms of a Fourier series (Navier's solution) and by summation of modes. First the homogeneous solution is given:

$$
w(x, y, t)=\sum_{n} \sum_{m} a_{m n}(t) \sin \left(\begin{array}{c}
m \pi x \\
a
\end{array}\right) \sin \left(\begin{array}{c}
m \pi y \\
b
\end{array}\right)
$$

The temporal equation is derived as:

$$
\ddot{a}_{m n}+a_{m n}\left[\left(\begin{array}{c}
m \pi \\
a
\end{array}\right)^{2}+\left(\begin{array}{c}
n \pi \\
b
\end{array}\right)^{2}\right]^{2} \begin{gathered}
D \\
\rho h
\end{gathered}=0 \text { where, } \omega_{m n}=\pi^{2} \sqrt{D}\left[\begin{array}{c}
m \\
\rho h
\end{array}\left[\begin{array}{c}
2 \\
a
\end{array}\right)^{2}+\left(\begin{array}{c}
n \\
b
\end{array}\right)^{2}\right]
$$

Let the solution of the temporal equation be the following:

$$
a_{m n}(t)=A_{m n} \sin \left(\omega_{m n} t+\phi\right) \text { where } A_{m n} \cos \phi=C_{m n} \text { and } A_{m n} \sin \phi=D_{m n}
$$

Thus:

$$
a_{m n}(t)=C_{m n} \sin \omega_{m n} t+D_{m n} \cos \omega_{m n} t
$$

Now the partial differential relationship of Heaviside functions and Dirac delta function is given by:

$$
\frac{\partial}{\partial x}\left(H\left(x-x_{1}\right)\right)=\delta\left(x-x_{1}\right) \text { and } \begin{gathered}
\partial \\
\partial x
\end{gathered}\left(\delta\left(x-x_{1}\right)\right)=\begin{gathered}
\delta\left(x-x_{1}\right) \\
\left(x-x_{1}\right)
\end{gathered}
$$

which implies:

$$
\begin{gathered}
\partial^{2} \\
\partial x^{2}
\end{gathered}\left(H\left(x-x_{1}\right)\right)=\begin{gathered}
\delta\left(x-x_{1}\right) \\
\left(x-x_{1}\right)
\end{gathered}
$$

Substituting Eq. (A9) into the RHS of Eq. (A3) we have:

$$
\begin{aligned}
\partial^{2} m_{x} & \begin{array}{r}
\partial^{2} m_{y} \\
\partial x^{2}
\end{array}=I_{1}+I_{2}=m_{x}^{1} \delta t\left[\begin{array}{cc}
\delta\left(x-x_{1}\right) & \delta\left(x-x_{2}\right) \\
x-x_{1} & x-x_{2}
\end{array}\right]\left[H\left(y-y_{1}\right)-H\left(y-y_{2}\right)\right]+ \\
& m_{y}^{1} \delta t\left[\begin{array}{cc}
\delta\left(y-y_{1}\right) & \delta\left(y-y_{2}\right) \\
y-y_{1} & y-y_{2}
\end{array}\right]\left[H\left(x-x_{1}\right)-H\left(x-x_{2}\right)\right]
\end{aligned}
$$

Now substituting Eqs (A4) (A7) and (A10) into Eq. (A3), then multiplying both sides by $\sin \left(\begin{array}{c}m^{\prime} \pi x \\ a\end{array}\right) \sin \left(\begin{array}{c}n^{\prime} \pi y \\ b\end{array}\right)$ and double integrating we get:

$$
\begin{aligned}
& \sum_{n} \sum_{m}\left(\rho h \ddot{a}_{m n}+a_{m n} \pi^{4}\left[\left(\begin{array}{c}
m \\
a
\end{array}\right)^{2}+\left(\begin{array}{l}
n \\
b
\end{array}\right)^{2}\right]^{2} D\right) \iint_{00}^{b a}\left(\sin \left(\begin{array}{c}
m \pi x \\
a
\end{array}\right) \sin \left(\begin{array}{c}
n \pi y \\
b
\end{array}\right) \sin \left(\begin{array}{c}
m^{\prime} \pi x \\
a
\end{array}\right) \sin \left(\begin{array}{c}
n^{\prime} \pi y \\
b
\end{array}\right) d x d y\right) \\
& \quad=\delta(t) \int_{0}^{b} \int_{0}^{a}\left(I_{1}+I_{2}\right) \sin \left(\begin{array}{c}
m^{\prime} \pi x \\
a
\end{array}\right) \sin \left(\begin{array}{c}
n^{\prime} \pi y \\
b
\end{array}\right) d x d y
\end{aligned}
$$


Using the property of orthogonalization, Eqs (9) and (10), and when $m=m^{\prime}$ and $n=n^{\prime}$, Eq. (A11) reduces to:

$$
\left(\ddot{a}_{m n}+a_{m n} \underset{\rho \pi^{4}}{D h}\left[\left(\begin{array}{c}
m \\
a
\end{array}\right)^{2}+\left(\begin{array}{c}
n \\
b
\end{array}\right)^{2}\right]^{2}\right)=\underset{\rho a b h}{4} \delta(t) \int_{0}^{b} \int_{0}^{a}\left(I_{1}+I_{2}\right) \sin \left(\begin{array}{c}
m^{\prime} \pi x \\
a
\end{array}\right) \sin \left(\begin{array}{c}
n^{\prime} \pi y \\
b
\end{array}\right) d x d y \text { (A12) }
$$

Eqnarray (A12) can be rewritten as:

$$
\left(\ddot{a}_{m n}+a_{m n} \omega_{m n}^{2}\right)=\underset{\rho a b h}{4} \delta(t)\left\{I_{3}+I_{4}\right\}
$$

where

$$
\begin{aligned}
I_{3} & =\int_{0}^{b} \int_{0}^{a} m_{x}^{1} \delta t\left[\begin{array}{cc}
\delta\left(x-x_{1}\right) & \delta\left(x-x_{2}\right) \\
x-x_{1} & x-x_{2}
\end{array}\right]\left[H\left(y-y_{1}\right)-H\left(y-y_{2}\right)\right] \sin \left(\begin{array}{c}
m^{\prime} \pi x \\
a
\end{array}\right) \sin \left(\begin{array}{c}
n^{\prime} \pi y \\
b
\end{array}\right) d x d y \text { (A14) } \\
I_{4} & =\int_{0}^{b} \int_{0}^{a} m_{y}^{1} \delta t\left[\begin{array}{cc}
\delta\left(y-y_{1}\right) & \delta\left(y-y_{2}\right) \\
y-y_{1} & y-y_{2}
\end{array}\right]\left[H\left(x-x_{1}\right)-H\left(x-x_{2}\right)\right] \sin \left(\begin{array}{c}
m^{\prime} \pi x \\
a
\end{array}\right) \sin \left(\begin{array}{c}
n^{\prime} \pi y \\
b
\end{array}\right) d x d y \text { (A15) }
\end{aligned}
$$

Mathematical definitions of Delta and Heaviside function states that:

$$
\delta\left(x-x_{1}\right)=\left\{\begin{array}{l}
=1 \text { when } x=x_{1} \\
=0 \text { when } x \neq x_{1}
\end{array}\right\} \text { and } H\left(x-x_{1}\right)=\left\{\begin{array}{l}
=1 \text { when } x \geqslant x_{1} \\
=0 \text { when } x<x_{1}
\end{array}\right\}
$$

The integral identity,

$$
\int_{a_{1}}^{a_{2}}\left\{\delta^{\prime}(x-a) f(x)\right\} d x=\left[-f^{\prime}(x)\right]_{x=a}
$$

where prime indicates the first derivative, is also used in this derivation.

Equation (A14) can be rewritten as:

$$
I_{3}=m_{x}^{1} \delta t \int_{0}^{b}\left[H\left(y-y_{1}\right)-H\left(y-y_{2}\right)\right] \sin \left(\begin{array}{c}
n^{\prime} \pi y \\
b
\end{array}\right) d y \int_{0}^{a}\left[\begin{array}{c}
\delta\left(x-x_{1}\right) \\
x-x_{1}-\delta\left(x-x_{2}\right) \\
x-x_{2}
\end{array}\right] \sin \left(\begin{array}{c}
m^{\prime} \pi x \\
a
\end{array}\right) d x
$$

Applying mathematical identities Eqs (A16) and (A17) in Eq. (A18) we get

$$
I_{3}=m_{x}^{1} \delta t(-1)\left(\begin{array}{c}
m^{\prime} b \\
n^{\prime} a
\end{array}\right)\left[\begin{array}{cc}
m^{\prime} \pi x_{1} & -\cos m^{\prime} \pi x_{2} \\
a & a
\end{array}\right]\left[\begin{array}{cc}
n^{\prime} \pi y_{1} & -\cos n^{\prime} \pi y_{2} \\
b & b
\end{array}\right]
$$

Similarly

$$
I_{4}=m_{y}^{1} \delta t(-1)\left(\begin{array}{c}
n^{\prime} a \\
m^{\prime} b
\end{array}\right)\left[\begin{array}{cc}
m^{\prime} \pi x_{1} & m^{\prime} \pi x_{2} \\
a & -\cos \\
\cos
\end{array}\right]\left[\begin{array}{cc}
n^{\prime} \pi y_{1} & n^{\prime} \pi y_{2} \\
b & -\cos \\
& b
\end{array}\right]
$$

Therefore Eq. (A14) can rewritten as the following when $m=m^{\prime}$ and $n=n^{\prime}$ :

$$
\left(\ddot{a}_{m n}+a_{m n} \omega_{m n}^{2}\right)=F_{m n} \delta(t)
$$

where

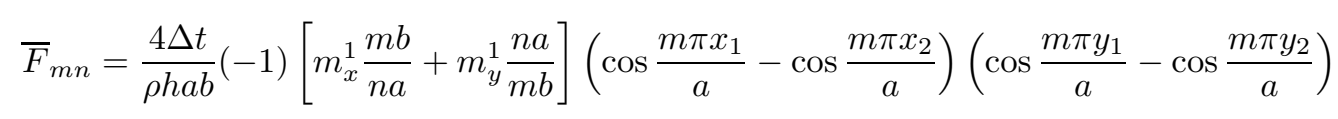


On adding modal damping to the temporal Eq. (A21), it becomes:

$$
\ddot{a}_{m n}+2 \zeta_{m n} \omega_{m n} \stackrel{\bullet}{m n}_{m}+\omega_{m n}^{2} a_{m n}=F_{m n} \delta(t)
$$

where the solution is given by

$$
a_{m n}(t)=\begin{aligned}
& F_{m n} \\
& \omega_{d_{m n}}
\end{aligned} e^{-\zeta_{m n} \omega_{m n} t} \sin \left(\omega_{d_{m n}} t\right)
$$

Thus the equation for transverse displacement as given by Eq. (A4) can be written as:

$$
w(x, y, t)=\sum_{n} \sum_{m} F_{m n} \omega_{d_{m n}} e^{-\zeta_{m n} \omega_{m n} t} \sin \left(\omega_{d_{m n}} t\right) \sin \left(\begin{array}{c}
m \pi x \\
a
\end{array}\right) \sin \left(\begin{array}{c}
n \pi y \\
b
\end{array}\right)
$$

The corresponding strains can be suitably expressed as given in Eqs (25-27). The voltage output can be derived as shown in Section 2.2. For details of sensor array outputs and continuous sensor outputs, please refer to ref [27]. The equations for a steady state sinusoidal excitation can be similarly derived.

Appendix B - Derivation of a Hsu-Nielsen source excitation on a simply supported plate

The equation of motion is:

$$
D \nabla^{4} w(x, y, t)=-\rho h \ddot{w}+f(x, y, t)
$$

where the step function force $f(x, y, t)=f_{0} U(t) \delta\left(x-x_{c}\right) \delta\left(y-y_{c}\right)$ gives the magnitude of the input excitation, $w$ is the z-displacement, $\rho$ is mass density and $\mathrm{h}$ is the plate thickness. The plate flexural rigidity is given as $D=\begin{gathered}E h^{3} \\ 12\left(1-\nu^{2}\right)\end{gathered}$, and $U(t)=0$ when $t \leqslant 0$ and $U(t)=1$ when $t>0$. The boundary conditions for a rectangular simply supported plate of length $a$ and width $b$ are defined as:

$$
w=0, \frac{\partial^{2} w}{\partial x^{2}}=0, \text { at } x=0, x=a, w=0, \frac{\partial^{2} w}{\partial y^{2}}=0, \quad y=0, y=b
$$

The bending and shear strains are:

$$
\varepsilon_{x}=-z_{\partial x^{2}}^{\partial^{2} w}, \varepsilon_{y}=-z \frac{\partial^{2} w}{\partial y^{2}}, \gamma_{x y}=-\partial z \begin{aligned}
& \partial^{2} w \\
& \partial x \partial y
\end{aligned}
$$

First, the homogeneous solution is obtained using $f(x, y, t)=0$. Let

$$
w(x, y, t)=\sum_{n} \sum_{m} a_{m n}(t) \sin \left(\begin{array}{c}
m \pi x \\
a
\end{array}\right) \sin \left(\begin{array}{c}
n \pi y \\
b
\end{array}\right)
$$

Substitution of Eq. (B4) into Eq. (B1) with $f(x, y, t)=0$ gives:

$$
\begin{aligned}
& D\left(\sum_{n} \sum_{m} a_{m n}\left(\begin{array}{c}
m \pi \\
a
\end{array}\right)^{4} \sin \left(\begin{array}{c}
m \pi x \\
a
\end{array}\right) \sin \left(\begin{array}{c}
n \pi y \\
b
\end{array}\right)\right)+2 D\left(\sum_{n} \sum_{m} a_{m n}\left(\begin{array}{c}
m \pi \\
a
\end{array}\right)^{2}\left(\begin{array}{c}
n \pi \\
b
\end{array}\right)^{2} \sin \left(\begin{array}{c}
m \pi x \\
a
\end{array}\right) \sin \left(\begin{array}{c}
n \pi y \\
b
\end{array}\right)\right) \\
& +D\left(\sum_{n} \sum_{m} a_{m n}\left(\begin{array}{c}
n \pi \\
a
\end{array}\right)^{4} \sin \left(\begin{array}{c}
m \pi x \\
a
\end{array}\right) \sin \left(\begin{array}{c}
n \pi y \\
b
\end{array}\right)\right)=-\rho h\left(\sum_{n} \sum_{m} \ddot{a}_{m n} \sin \left(\begin{array}{c}
m \pi x \\
a
\end{array}\right) \sin \left(\begin{array}{c}
n \pi y \\
b
\end{array}\right)\right)(\mathrm{B} 5)
\end{aligned}
$$

or,

$$
\begin{aligned}
& D\left(\sum_{n} \sum_{m} a_{m n}\left[\left(\begin{array}{c}
m \pi \\
a
\end{array}\right)^{2}+\left(\begin{array}{c}
n \pi \\
b
\end{array}\right)^{2}\right]^{2} \sin \left(\begin{array}{c}
m \pi x \\
a
\end{array}\right) \sin \left(\begin{array}{c}
n \pi y \\
b
\end{array}\right)\right)=-\rho h\left(\sum_{n} \sum_{m} \ddot{a}_{m n} \sin \left(\begin{array}{c}
m \pi x \\
a
\end{array}\right) \sin \left(\begin{array}{c}
n \pi y \\
b
\end{array}\right)\right)(\mathrm{B} 6) \\
& \left.\sum_{n} \sum_{m}\left(\rho h \ddot{a}_{m n}+a_{m n}\left[\left(\begin{array}{c}
m \pi \\
a
\end{array}\right)^{2}+\left(\begin{array}{c}
n \pi \\
b
\end{array}\right)^{2}\right]^{2} D\right) \sin \left(\begin{array}{c}
m \pi x \\
a
\end{array}\right) \sin \left(\begin{array}{c}
n \pi y \\
b
\end{array}\right)\right)=0
\end{aligned}
$$


Multiplying both sides by $\sin _{a}^{m^{\prime} \pi x} \sin _{b}^{n^{\prime} \pi y}$ and doubly integrating:

$$
\begin{aligned}
& \sum_{n} \sum_{m}\left(\rho h \ddot{a}_{m n}+a_{m n}\left[\left(\begin{array}{c}
m \pi \\
a
\end{array}\right)^{2}+\left(\begin{array}{c}
n \pi \\
b
\end{array}\right)^{2}\right]^{2} D\right) \int_{0}^{b} \int_{0}^{a} \sin \left(\begin{array}{c}
m^{\prime} \pi x \\
a
\end{array}\right) \sin \left(\begin{array}{c}
n^{\prime} \pi y \\
b
\end{array}\right) \\
& \sin \left(\begin{array}{c}
m \pi x \\
a
\end{array}\right) \sin \left(\begin{array}{c}
n \pi y \\
b
\end{array}\right) d x d y=0
\end{aligned}
$$

When $m^{\prime}=m, n^{\prime}=n$

$$
\int_{0}^{b} \int_{0}^{a} \sin \left(\begin{array}{c}
m^{\prime} \pi x \\
a
\end{array}\right) \sin \left(\begin{array}{c}
n^{\prime} \pi y \\
b
\end{array}\right) \sin \left(\begin{array}{c}
m \pi x \\
a
\end{array}\right) \sin \left(\begin{array}{c}
n \pi y \\
b
\end{array}\right) d x d y=\begin{gathered}
a b \\
4
\end{gathered}
$$

and when $m^{\prime} \neq m, n^{\prime} \neq n, m^{\prime}=m, n^{\prime} \neq n$, and $m^{\prime} \neq m, n^{\prime}=n$

$$
\int_{0}^{b} \int_{0}^{a} \sin \left(\begin{array}{c}
m^{\prime} \pi x \\
a
\end{array}\right) \sin \left(\begin{array}{c}
n^{\prime} \pi y \\
b
\end{array}\right) \sin \left(\begin{array}{c}
m \pi x \\
a
\end{array}\right) \sin \left(\begin{array}{c}
n \pi y \\
b
\end{array}\right) d x d y=0
$$

Only the $\mathrm{m}, \mathrm{n}^{\text {th }}$ term remains and since the integral cannot be always zero, therefore,

$$
\ddot{a}_{m n}+a_{m n}\left[\left(\begin{array}{c}
m \pi \\
a
\end{array}\right)^{2}+\left(\begin{array}{c}
n \pi \\
b
\end{array}\right)^{2}\right]_{\rho h}^{2}=0
$$

This represents a linear system with a natural frequency given by:

$$
\omega_{m n}=\pi^{2} \sqrt{D} \begin{gathered}
D \\
\rho h
\end{gathered}\left[\left(\begin{array}{c}
m \\
a
\end{array}\right)^{2}+\left(\begin{array}{c}
n \\
b
\end{array}\right)^{2}\right]
$$

The forced response problem can be written as:

$$
D \nabla^{4} w+\rho h \ddot{w}=f_{0} U(t) \delta\left(x-x_{c}\right) \delta\left(y-y_{c}\right)
$$

Substituting Eq. (B4) into Eq. (B13) gives:

$$
\begin{aligned}
& \left.\sum_{m} \sum_{n}\left(\rho h \ddot{a}_{m n}+a_{m n} \pi^{4}\left[\left(\begin{array}{c}
m \\
a
\end{array}\right)^{2}+\left(\begin{array}{l}
n \\
b
\end{array}\right)^{2}\right]^{2} D\right) \sin \left(\begin{array}{c}
m \pi x \\
a
\end{array}\right) \sin \left(\begin{array}{c}
n \pi y \\
b
\end{array}\right)\right) \\
& =f_{0} U(t)\left[\delta\left(x-x_{c}\right) \delta\left(y-y_{c}\right)\right]
\end{aligned}
$$

Multiplying both sides of Eq. (B14) by $\sin \left(\begin{array}{c}m^{\prime} \pi x \\ a\end{array}\right) \sin \left(\begin{array}{c}n^{\prime} \pi y \\ b\end{array}\right)$ and double integrating:

$$
\begin{aligned}
& \sum_{n} \sum_{m}\left(\rho h \ddot{a}_{m n}+a_{m n} \pi^{4}\left[\left(\begin{array}{c}
m \\
a
\end{array}\right)^{2}+\left(\begin{array}{l}
n \\
b
\end{array}\right)^{2}\right]^{2} D\right) \int_{0}^{b} \int_{0}^{a}\left(\sin \left(\begin{array}{c}
m \pi x \\
a
\end{array}\right) \sin \left(\begin{array}{c}
n \pi y \\
b
\end{array}\right) \sin \left(\begin{array}{c}
m^{\prime} \pi x \\
a
\end{array}\right)\right. \\
& \left.\sin \left(\begin{array}{c}
n^{\prime} \pi y \\
b
\end{array}\right) d x d y\right)=f_{0} U(t) \int_{0}^{b} \int_{0}^{a} \delta\left(x-x_{c}\right) \delta\left(y-y_{c}\right) \sin \left(\begin{array}{c}
m^{\prime} \pi x \\
a
\end{array}\right) \sin \left(\begin{array}{c}
n^{\prime} \pi y \\
b
\end{array}\right) d x d y
\end{aligned}
$$

when $m=m^{\prime}$ and $n=n^{\prime}$, the left hand side becomes nonzero:

$$
\left(\rho h \ddot{a}_{m n}+a_{m n} \pi^{4}\left[\left(\begin{array}{c}
m \\
a
\end{array}\right)^{2}+\left(\begin{array}{c}
n \\
b
\end{array}\right)^{2}\right]^{2} D\right)_{4}^{a b}=f_{0} U(t) \sin \left(\begin{array}{c}
m^{\prime} \pi x_{c} \\
a
\end{array}\right) \sin \left(\begin{array}{c}
n^{\prime} \pi y_{c} \\
b
\end{array}\right)
$$




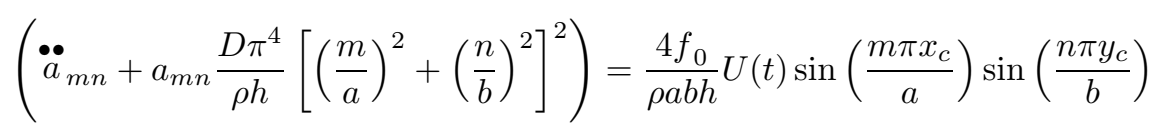

From Eq. (B12)

$$
\begin{aligned}
\omega_{m n}^{2} & =\begin{array}{c}
D \pi^{4} \\
\rho h
\end{array}\left[\left(\begin{array}{c}
m \\
a
\end{array}\right)^{2}+\left(\begin{array}{l}
n \\
b
\end{array}\right)^{2}\right]^{2} . \text { Let } \\
F_{m n} & =\begin{array}{c}
4 f_{0} \\
\rho h a b
\end{array} \sin \left(\begin{array}{c}
m \pi x_{c} \\
a
\end{array}\right) \sin \left(\begin{array}{c}
n \pi y_{c} \\
b
\end{array}\right) .
\end{aligned}
$$

Addition of modal damping to the temporal equation, where each damping ratio for each of the natural frequencies is specified independently, yields:

$$
\ddot{a}_{m n}+2 \zeta_{m n} \omega_{m n} \dot{a}_{m n}+\omega_{m n}^{2} a_{m n}=F(t)
$$

where $F(t)=F_{m n} U(t)$ and $a_{m n}$ is the modal participating factor. Assuming initial conditions initial displacement and velocity, to be zeros, and using convolution integrals [30], the time response is obtained as:

$$
a_{m n}(t)=\omega_{d_{m n}}^{1} e^{-\zeta_{m n} \omega_{m n} t}\left[\int_{-t}^{0}(0) e^{-\zeta_{m n} \omega_{m n} t} \sin \omega_{d}(t-\tau) d \tau+\int_{0}^{t} F_{m n} e^{-\zeta_{m n} \omega_{m n} t} \sin \omega_{d}(t-\tau) d \tau\right]
$$

or,

$$
a_{m n}(t)=F_{m n} e_{d_{m n}} e^{-\zeta_{m n} \omega_{m n} t}\left[\int_{0}^{t} e^{-\zeta_{m n} \omega_{m n} \tau} \sin \omega_{d}(t-\tau) d \tau\right]
$$

and if $t(0)=0$,

$$
a_{m n}(t)=\begin{aligned}
& F_{m n} \\
& \omega_{m n}^{2}-F_{m n}^{2} \sqrt{ } 1-\zeta^{2}
\end{aligned} e^{-\zeta_{m n} \omega_{m n}(t-t(0))} \cos \left[\omega_{d}(t-t(0))-\theta\right]
$$

Therefore the solution can be written as:

$$
a_{m n}(t)=\begin{aligned}
& F_{m n} \\
& \omega_{m n}^{2}
\end{aligned}-\left(\begin{array}{c}
F_{m n} \\
\omega_{m n} \omega_{d_{m n}}
\end{array} e^{-\zeta_{m n} \omega_{m n} t} \cos \left(\omega_{d_{m n}} t-\theta\right)\right)
$$

when $t>0, \theta=\tan ^{-1}\left(\begin{array}{c}\zeta \\ \sqrt{ } 1-\zeta^{2}\end{array}\right), \omega_{d_{m n}}=\omega_{m n} \sqrt{ } 1-\zeta_{m n}^{2}$ and $\omega_{m n}=\pi^{2} \sqrt{\rho} \begin{gathered}D \\ \rho h\end{gathered}\left[\left(\begin{array}{c}m \\ a\end{array}\right)^{2}+\left(\begin{array}{c}n \\ b\end{array}\right)^{2}\right]$. Thus the total solution for the plate displacement can be expressed as:

$$
w(x, y, t)=\sum_{n} \sum_{m}\left(\begin{array}{c}
F_{m n} \\
\omega_{m n}^{2}
\end{array}-\left(\begin{array}{c}
F_{m n} \\
\omega_{m n} \omega_{d_{m n}}
\end{array} e^{-\zeta_{m n} \omega_{m n} t} \cos \left(\omega_{d_{m n}} t-\theta\right)\right)\right) \sin \left(\begin{array}{c}
m \pi x \\
a
\end{array}\right) \sin \left(\begin{array}{c}
n \pi y \\
b
\end{array}\right)
$$

The damping ratio is $\zeta_{m n}$ and can be specified individually for each mode. For zero damping, i.e., when $\zeta=0$,

$$
w(x, y, t)=\sum_{n} \sum_{m} F_{m n}^{2}\left(1-\cos \left(\omega_{m n} t\right)\right) \sin \left(\begin{array}{c}
m \pi x \\
a
\end{array}\right) \sin \left(\begin{array}{c}
n \pi y \\
b
\end{array}\right)
$$




\section{References}

[1] S.G. Pierce, B. Culshaw, G. Manson, K. Worden and W.J. Staszewski, The application of ultrasonic Lamb wave techniques to the evaluation of advanced composite structures, Procs. of SPIE 3986 (2000), 93-103.

[2] D.N. Allyne and P. Cawley, Optimization of Lamb wave inspection techniques, J. Destructive Testing and Evaluation International 25(Issue 1) (1992), 11-22.

[3] S.G. Pierce, W.R. Phillip, B. Culshaw, A. Gachagan, A. McNab, G. Hayward and F. Lecuyer, Surface-bonded optical sensors for the inspection of CFRP plates using ultrasonic Lamb waves, Smart Materials and Structures 5 (1996), 776-787.

[4] A. Gachagan, G. Hayward, A. McNab, P. Reynolds, S.G. Pierce, W.R. Phillip and B. Culshaw, Generation and Reception of Ultrasonic guided waves in composite plates using conformable Piezoelectric Transmitters and Optical-Fiber detectors, IEEE Trans. on Ultrasonics, Ferroelectrics, and Frequency Control 46(1) (1999), 72-81.

[5] J.L. Rose, Guided Wave Nuances for Ultrasonic Nondestructive Evaluation, IEEE Trans. on Ultrasonics, Ferroelectrics, and Frequency Control 47(3) (May), 575-582.

[6] C. Wang and F. Chang, Diagnosis of Impact Damage in Composite Structures with Built-In Piezoelectrics Network, Proceedings of the SPIE 3990 (2000), 13.

[7] W.G. Schwartz, M.E. Read, M.J. Kremer, M.K. Hinders and B.T. Smith, Lamb Wave Tomographic Imaging System for Aircraft Structural Health Assessment, SPIE Conference on NDE of Aging Aircraft, Airports, and Aerospace Hardware III (Vol. 3586) (1999), p. 292, Newport Beach, CA.

[8] M.J. Sundaresan, A. Ghoshal, M.J. Schulz and C. Wilkerson, Acoustic Emission Monitoring using Distributed Sensors, ASNT Spring Conference and 9th Annual Research Symposium, Birmingham, Alablama, March 27-31, 2000.

[9] M.J. Sundaresan, M.J. Schulz, A. Ghoshal and P. Pratap, A Neural System for Structural Health Monitoring, SPIE Sh $^{\text {th }}$ Int. Symposium on Smart Materials and Structures, March 4-8, 2001.

[10] M.J. Sundaresan, A. Ghoshal and M.J. Schulz, Sensor Array System, Patent No. 6,399,939 B1, US Patent Office, June 4, 2002.

[11] A. Ghoshal, M.J. Sundaresan, M.J. Schulz and P.F. Pai, Continuous Sensors for Structural Health Monitoring, Adaptive Structures and Material Systems Symposium at the International Mechanical Engineering Congress and Exposition Winter Annual Meeting of the ASME, Nov. 5-10, 2000, Walt Disney World Dolphin, Orlando, Fla.

[12] M.J. Schulz, M.J. Sundaresan, A. Ghoshal and W.N. Martin, Evaluation of Distributed Sensors for Structural Health Monitoring, ASME DTEC'01 Conf., Sept. 9-12, Pittsburgh, PA, 2001.

[13] J.F. Doyle, Wave Propagation in Structures, $2^{\text {nd }}$ Ed., Springer-Verlag, NY, 1997.

[14] J.D. Achenbach, Wave Propagation in Elastic Solids, North Holland Publishing Company, 1973.

[15] D.S. Ballentine, R.M. White, S.J. Martin, A.J. Ricco, E.T. Zellers, G.C. Frye and H. Wohltjen, Acoustic Wave Sensors, Theory, Design and Physico-Chemical Applications, Academic Press Inc, 1997.

[16] C.K. Campbell, Surface Acoustic Wave Devices for Mobile and Wireless Communications, Academic Press, 1997.

[17] L.W. Schmerr, Jr., Fundamentals of Ultrasonic Nondestructive Evaluation, Plenum Press, 1998.

[18] R.J. Wasley, Stress Wave Propagation in Solids, Marcel Dekker, Inc, New York, 1973.

[19] M.R. Gorman and W.H. Prosser, Application of Normal Mode Expansion to AE waves in Finite Plates, Transactions of the ASME: Journal of Applied Mechanics 63(2) (1996), 555-557.

[20] W.H. Prosser, The propagation characteristics of the Plate Modes of Acoustic Emission Waves in thin Aluminum Plates and Thin Graphite/Epoxy Composites Plates and Tubes, Ph.D. dissertation, The Johns Hopkins University, Baltimore, Maryland, 1991.

[21] M.A. Hamstad, J. Gary and A. O'Gallagher, Far Field Acoustic Emission Waves by Three Dimensional Finite Element Modeling of Pencil-Lead Breaks on a Thick Plate, Journal of Acoustic Emission 14(2) (1996), 103-114.

[22] M. Johnson and P. Gudmundson, Experimental and Theoretical Characterization of Acoustic Emission Transients in Composites Laminates, Composites Science and Technology 61 (2001), 1367-1378.

[23] E.K. Dimitriadis, C.R. Fuller and C.A. Rogers, Piezoelectric Actuators for Distributed Vibration Excitation of Thin Plates, ASME Journal of Vibration and Acoustics 113 (January 1991), 100-107.

[24] V.R. Sonti, S.J. Kim and J.D. Jones, Equivalent Forces and Wavenumber Spectra of Shaped Piezoelectric Actuators, Journal of Sound and Vibration 187(1) (1995), 111-131.

[25] C.K. Lee, Theory of Laminated Piezoelectric Plates for the Design of Distributed Sensors/Actuators. Part I: Governing Equations and Reciprocal Relationships, Journal of Acoustical Society of America 87(3) (March 1990), 1144-1158.

[26] C.Y.K. Chee, L. Tong and G. Steven, A review on the Modeling of Piezoelectric Sensors and Actuators Incorporated in Intelligent Structures, Journal of Intelligent Materials and Structures 9 (January 1998), Technomic Publishing Co., Inc., Lancaster, PA 17604.

[27] W.N. Martin Jr., A. Ghoshal, M.J. Sundaresan, M.J. Schulz and G. Lebby, An Artificial Nerve sensing for Structural Health Monitoring, SPIE Conference 2002, San Diego, CA, Mar. 17-21, 2002, pp. \# 4702-4706.

[28] S.P. Timoshenko and S. Wonoisky-Krieger, Theory of Plates \& Shells, 2nd ed., The McGraw-Hill Companies, 1959.

[29] A.C. Ugural, Stresses in Plates and Shells, McGraw-Hill, 1981.

[30] D.J. Inman, Engineering Vibration, Prentice Hall, 2001.

[31] W.N. Martin, An Artificial Neural System for Structural Health Monitoring, Ph.D. Dissertation, North Carolina A\&T State University, Greensboro, North Carolina, 2001.

[32] Goutham R. Kirikera, An Artificial Neural System for Distributed Sensing, MS thesis, University of Cincinnati, August 2003.

[33] A. Ghoshal, W.N. Martin Jr., M.J. Schulz, A. Chattopadhyay and W.H. Prosser, Wave Propagation Sensing for Damage Detection in Plates, 9th SPIE Conference 2002, San Diego, CA, Mar. 17-21, 2002, pp. \# SS 4693-4636.

[34] G.R. Kirikera, V. Shinde, M.J. Schulz, S. Datta, D. Hurd, M. Sundaresan and A. Ghoshal, Mimicking the biological neural system using electronic logic circuits, SPIE Smart Structures and NDE Conference, March 2004, California. 
[35] Invention Disclosure, Univ. of Cincinnati, 8-26-03, An Artificial Neural System, Goutham Kirikera, Saurabh Datta, Bo Westheider, Mark J. Schulz, Mannur J. Sundaresan, William N. Martin, Jr., Anindya Ghoshal, UC 103-046. 

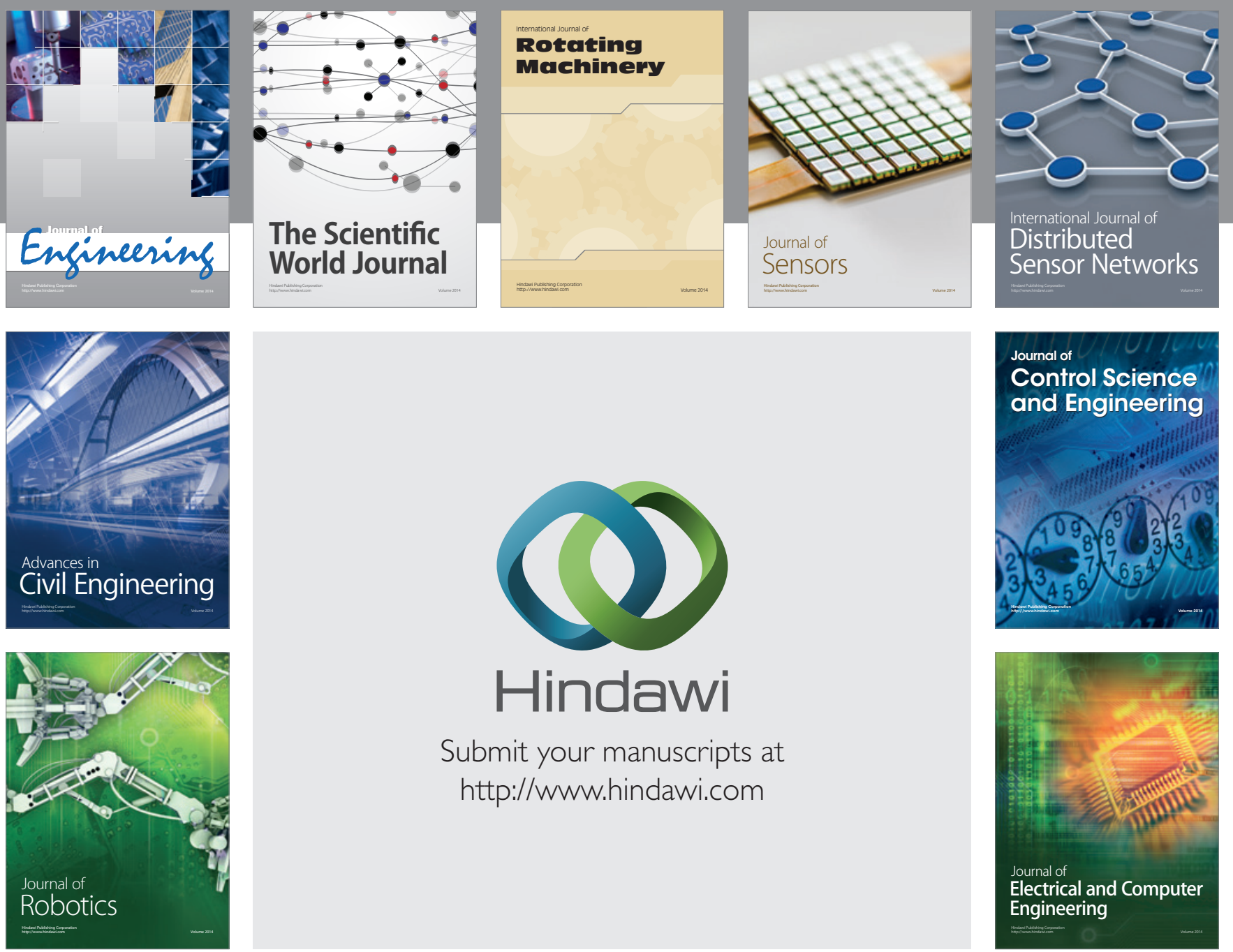

Submit your manuscripts at

http://www.hindawi.com
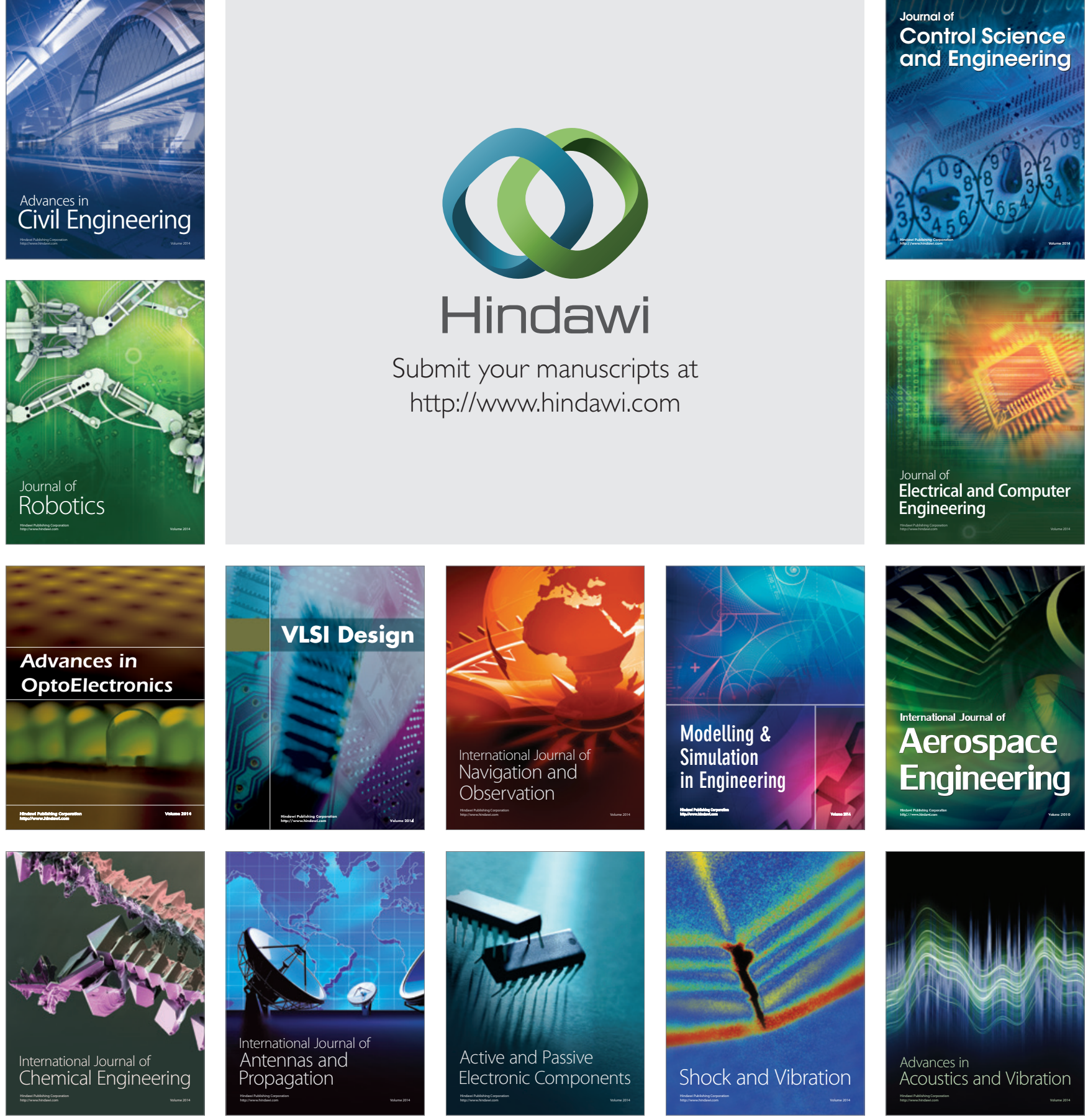